\title{
ExpTime Tableaux with Global Caching for Hybrid PDL
}

\author{
Linh Anh Nguyen \\ Institute of Informatics, University of Warsaw \\ Banacha 2, 02-097 Warsaw, Poland \\ nguyen@mimuw.edu.pl \\ July 30,2018
}

\begin{abstract}
We present the first direct tableau decision procedure with the ExpTime complexity for HPDL (Hybrid Propositional Dynamic Logic). It checks whether a given ABox (a finite set of assertions) in HPDL is satisfiable. Technically, it combines global caching with checking fulfillment of eventualities and dealing with nominals. Our procedure contains enough details for direct implementation and has been implemented for the TGC2 (Tableaux with Global Caching) system. As HPDL can be used as a description logic for representing and reasoning about terminological knowledge, our procedure is useful for practical applications.
\end{abstract}

\section{Introduction}

Propositional dynamic logic (PDL) $[58$ is one of the most well-known modal logics. It was designed for reasoning about correctness of programs, but can be modified or extended for other purposes. For example, its extension $\mathrm{CPDL}_{r e g}$ with converse and regular inclusion axioms can be used as a framework for multi-agent logics [4]. As another example, the description logic $\mathcal{A L C}_{\text {reg }}$ is a variant of PDL for representing and reasoning about terminological knowledge [17. Extensions of $\mathcal{A L C}_{\text {reg }}$ were also studied by researchers, including the ones with regular inclusion axioms, inverse roles, qualified number restrictions and nominals.

Automated reasoning in PDL and its extensions is useful for practical applications. The first tableau decision procedure for PDL was developed by Pratt [15]. It implicitly uses global caching and has the ExPTIME (optimal) complexity. Nguyen and Szałas [14] reformulated that procedure by explicitly using global caching and extended it for dealing with checking satisfiability of an ABox (a finite set of assertions) in PDL. Abate et al. [1 gave another tableau decision procedure with global caching for PDL, which updates fulfillment of eventualities (i.e., existential star modalities) on-the-fly. Due to global caching, the procedures given in [114] have the ExPTIME complexity.

Tableaux with global caching were formally formulated by Goré and Nguyen for the description logic $\mathcal{A L C}$ [6] and extended for other logics. A tableau with global caching for checking satisfiability of a concept w.r.t. a TBox in $\mathcal{A L C}$ is a rooted "andor" graph, where the label of each node is a set of concepts treated as requirements to be realized for the node and each edge departing from an "and"-node is labeled by a set of roles 1 Using global caching, each node has a unique label, which means that before creating a new node we check whether there already exists a node with the same label that can be used as a proxy. It is sufficient to deterministically construct one "and-or" graph and update the statuses of the nodes by detecting direct clashes and

\footnotetext{
${ }^{1}$ A concept is like a formula and a role is like an atomic program in PDL.
} 
propagating them backward appropriately. Extending tableaux with global caching for PDL [114], the ability to check fulfillment of eventualities over the constructed "and-or" graph is essential. Dealing with ABoxes in PDL [14], an "and-or" graph has two kinds of nodes: complex nodes and simple nodes. The label of a complex node is an ABox (with assertions about different states and accessibility between them), while the label of a simple node is a set of formulas (about one state). Edges departing from complex "and"-nodes lead to simple nodes and are labeled with more information.

HPDL (Hybrid PDL) is the modal logic that extends PDL with nominals. It is more expressive than PDL and belongs to the same complexity class ExPTimE-complete as PDL [516] (regarding the satisfiability problem). Kaminski and Smolka [9] developed a decision procedure for HPDL. The authors used the term "goal-directed" to refer to the property that the search is done analytically (i.e., closely based on the input). Like the traditional tableau method for description logics, the search space used in [9] is an "or"-tree of nodes that are "and"-structures, where the "or"-tree is generated by nondeterministic choices (using backtracking) and the "and"-structures are demo graphs (like model graphs or Hintikka structures without statically reduced assertions). Nodes of a demo graph, called "normal clauses" in [9], are formula sets without statically reduced formulas. Caching is done only within an "and"-structure. This is similar to the technique used in Donini and Massacci's tableau algorithm for the description logic $\mathcal{A L C}[3]$ and somehow equivalent to the "anywhere blocking" technique used in the traditional tableau method for description logics 2]. Without a surprise, the decision procedure given in 9] for HPDL has the NExPTime (non-optimal) complexity for the worst case. Devising efficient ExPTime decision procedures for HPDL and its extensions is claimed in [9] as an open problem.

In this article, we present the first tableau decision procedure with the ExPTime complexity for checking whether a given ABox (a finite set of assertions) in HPDL is satisfiable. Technically, it combines global caching with checking fulfillment of eventualities and the technique of dealing with nominals from our work on tableaux for the description logic $\mathcal{S H I O}$ [11. Global caching not only guarantees the ExPTIME complexity, but is also an important optimization technique for increasing efficiency. Our decision procedure for HPDL also uses other advanced techniques, for example, automaton-modal operators.

In the absence of nominals or the ability to express global assumptions, the problem of checking satisfiability of an ABox is usually more general than the problem of checking satisfiability of a formula. In HPDL, the former problem is reducible to the latter. We consider the former instead of the latter due to the nature of our tableau method (which uses both complex nodes and simple nodes for tableaux).

Our decision procedure for HPDL contains enough details for direct implementation and has been implemented for TGC2 [12, which is a system based on tableaux with global caching for automated reasoning in modal and description logics. This system was designed and implemented with several optimization techniques. Regarding memory management, experiments showed that the amount of memory used by TGC2 is competitive with the ones used by the other reasoners. As far as we know, TGC2 is the first implemented system that can be used for reasoning in HPDL. We refer the reader to 12 for details of the design of this system.

The rest of this article is structured as follows. In Section 2, we present the syntax and semantics of HPDL and recall automaton-modal operators [8/4. We omit the feature of "global assumptions" as they can be expressed in PDL (by "local assump- 
tions"). In Section 3, we present our tableau calculus for HPDL, starting with the data structure, the tableau rules and ending with the corresponding tableau decision procedure and its properties. In Section 4, we present an example to illustrate our procedure. We estimate the complexity of the procedure and prove its soundness and completeness in Section 5. Concluding remarks are given in Section 6,

\section{Preliminaries}

\subsection{Hybrid Propositional Dynamic Logic}

We use $\Sigma$ to denote the set of atomic programs, $\mathcal{P R O P}$ to denote the set of propositions (i.e., atomic formulas), and $\mathcal{O}$ to denote the set of nominals. We denote elements of $\Sigma$ by letters like $\sigma$ and $\varrho$, elements of $\mathcal{P R O P}$ by letters like $p$ and $q$, and elements of $\mathcal{O}$ by letters like $a$ and $b$.

A Kripke model is a pair $\mathcal{M}=\left(\Delta^{\mathcal{M}},{ }^{\mathcal{M}}\right)$, where $\Delta^{\mathcal{M}}$ is a set of states and $\cdot \mathcal{M}$ is an interpretation function that maps each nominal $a \in \mathcal{O}$ to an element $a^{\mathcal{M}}$ of $\Delta^{\mathcal{M}}$, each proposition $p \in \mathcal{P} \mathcal{R O P}$ to a subset $p^{\mathcal{M}}$ of $\Delta^{\mathcal{M}}$ and each atomic program $\sigma \in \Sigma$ to a binary relation $\sigma^{\mathcal{M}}$ on $\Delta^{\mathcal{M}}$. Intuitively, $p^{\mathcal{M}}$ is the set of states in which $p$ is true and $\sigma^{\mathcal{M}}$ is the binary relation consisting of pairs (inputstate, outputstate) of the program $\sigma$.

Formulas and programs of the base language of HPDL are defined by the following grammar rules, respectively, where $p \in \mathcal{P} \mathcal{R O P}$, $a \in \mathcal{O}$ and $\sigma \in \Sigma$ :

$$
\begin{aligned}
& \varphi::=\top|\perp| p|a| \neg \varphi|\varphi \wedge \varphi| \varphi \vee \varphi|\varphi \rightarrow \varphi|\langle\alpha\rangle \varphi \mid[\alpha] \varphi \\
& \alpha::=\sigma|\alpha ; \alpha| \alpha \cup \alpha\left|\alpha^{*}\right| \varphi ?
\end{aligned}
$$

We use letters like $\alpha, \beta$ to denote programs and letters like $\varphi, \psi, \chi$ to denote formulas. The intended meaning of program operators is as follows:

$-\alpha ; \beta$ stands for the sequential composition of $\alpha$ and $\beta$,

$-\alpha \cup \beta$ stands for the non-deterministic choice between $\alpha$ and $\beta$,

- $\alpha^{*}$ stands for the repetition of $\alpha$ a non-deterministic number of times,

$-\varphi$ ? stands for checking whether $\varphi$ holds for the current state.

Informally, a formula $\langle\alpha\rangle \varphi$ represents the set of states $x$ such that the program $\alpha$ has a transition from $x$ to a state $y$ satisfying $\varphi$. Dually, a formula $[\alpha] \varphi$ represents the set of states $x$ from which every transition of $\alpha$ leads to a state satisfying $\varphi$. A formula $a$ (a nominal) represents the set consisting of the only state specified by $a$.

Formally, the interpretation function of a Kripke model $\mathcal{M}$ is extended to interpret complex formulas and complex programs as shown in Figure1. For a set $\Gamma$ of formulas, we denote $\Gamma^{\mathcal{M}}=\bigcap\left\{\varphi^{\mathcal{M}} \mid \varphi \in \Gamma\right\}$. If $w \in \varphi^{\mathcal{M}}$ (resp. $w \in \Gamma^{\mathcal{M}}$ ), then we say that $\varphi$ (resp. $\Gamma$ ) is satisfied at $w$ in $\mathcal{M}$. If there exists a Kripke model $\mathcal{M}$ such that $\varphi^{\mathcal{M}}$ (resp. $\Gamma^{\mathcal{M}}$ ) is not empty, then $\varphi$ (resp. $\Gamma$ ) is satisfiable.

An assertion is an expression of the form $a: \varphi$ or $\sigma(a, b)$. An $A B o x$ is a finite set of assertions. Let null: $\varphi$ stand for $\varphi$. By letters like $o, o_{1}, o_{2}$ we will denote nominals or null, and by letters like $\xi, \zeta$ we will denote formulas or assertions.

We define:

$$
\begin{aligned}
& \mathcal{M} \models a: \varphi \quad \text { iff } \quad a^{\mathcal{M}} \in \varphi^{\mathcal{M}}, \\
& \mathcal{M} \models \sigma(a, b) \quad \text { iff } \quad\left(a^{\mathcal{M}}, b^{\mathcal{M}}\right) \in \sigma^{\mathcal{M}} \text {. }
\end{aligned}
$$




$$
\begin{array}{rlrl}
(\alpha ; \beta)^{\mathcal{M}} & =\alpha^{\mathcal{M}} \circ \beta^{\mathcal{M}} & (\alpha \cup \beta)^{\mathcal{M}} & =\alpha^{\mathcal{M}} \cup \beta^{\mathcal{M}} \\
\left(\alpha^{*}\right)^{\mathcal{M}} & =\left(\alpha^{\mathcal{M}}\right)^{*} & (\varphi ?)^{\mathcal{M}} & =\left\{(x, x) \mid x \in \varphi^{\mathcal{M}}\right\} \\
T^{\mathcal{M}} & =\Delta^{\mathcal{M}} \perp^{\mathcal{M}}=\emptyset & a^{\mathcal{M}} & =\left\{a^{\mathcal{M}}\right\} \\
(\neg \varphi)^{\mathcal{M}} & =\Delta^{\mathcal{M}} \backslash \varphi^{\mathcal{M}} & (\varphi \rightarrow \psi)^{\mathcal{M}} & =(\neg \varphi \vee \psi)^{\mathcal{M}} \\
(\varphi \wedge \psi)^{\mathcal{M}} & =\varphi^{\mathcal{M}} \cap \psi^{\mathcal{M}} & (\varphi \vee \psi)^{\mathcal{M}} & =\varphi^{\mathcal{M}} \cup \psi^{\mathcal{M}} \\
(\langle\alpha\rangle \varphi)^{\mathcal{M}} & =\left\{x \in \Delta^{\mathcal{M}} \mid \exists y\left((x, y) \in \alpha^{\mathcal{M}} \wedge y \in \varphi^{\mathcal{M}}\right)\right\} \\
([\alpha] \varphi)^{\mathcal{M}} & =\left\{x \in \Delta^{\mathcal{M}} \mid \forall y\left((x, y) \in \alpha^{\mathcal{M}} \rightarrow y \in \varphi^{\mathcal{M}}\right)\right\}
\end{array}
$$

Fig. 1. Interpretation of complex programs and complex formulas.

If $\mathcal{M} \models \xi$, then we say that $\mathcal{M}$ satisfies $\xi$. We say that $\mathcal{M}$ satisfies and is a model of an $\mathrm{ABox} \Gamma$, and $\Gamma$ is satisfied in $\mathcal{M}$, denoted by $\mathcal{M} \models \Gamma$, if $\mathcal{M}$ satisfies all assertions in $\Gamma$. If $\Gamma$ is satisfied in some Kripke model $\mathcal{M}$, then it is satisfiable.

Formulas $\varphi$ and $\psi$ are equivalent, denoted by $\varphi \equiv \psi$, if $\varphi^{\mathcal{M}}=\psi^{\mathcal{M}}$ for every Kripke model $\mathcal{M}$. Assertions $\xi$ and $\zeta$ are equivalent, denoted $\xi \equiv \zeta$, if for every Kripke model $\mathcal{M}, \mathcal{M} \models \xi$ iff $\mathcal{M} \models \zeta$.

A formula/assertion is in the negation normal form (NNF) if it does not use $\rightarrow$ and it uses $\neg$ only immediately before propositions or nominals. Every formula/assertion can be translated in polynomial time to an equivalent formula/assertion in NNF. From now on, by $\bar{\varphi}$ we denote the NNF of $\neg \varphi$. For an assertion $\xi=a: \varphi$, by $\bar{\xi}$ we denote $a: \bar{\varphi}$. An ABox is in NNF if all of its assertions are in NNF.

\subsection{Automaton-Modal Operators}

The alphabet $\Sigma(\alpha)$ of a program $\alpha$ and the regular language $\mathcal{L}(\alpha)$ generated by $\alpha$ are specified as follows 2

$$
\begin{array}{ll}
\Sigma(\sigma)=\{\sigma\} & \mathcal{L}(\sigma)=\{\sigma\} \\
\Sigma(\varphi ?)=\{\varphi ?\} & \mathcal{L}(\varphi ?)=\{\varphi ?\} \\
\Sigma(\beta ; \gamma)=\Sigma(\beta) \cup \Sigma(\gamma) & \mathcal{L}(\beta ; \gamma)=\mathcal{L}(\beta) \cdot \mathcal{L}(\gamma) \\
\Sigma(\beta \cup \gamma)=\Sigma(\beta) \cup \Sigma(\gamma) & \mathcal{L}(\beta \cup \gamma)=\mathcal{L}(\beta) \cup \mathcal{L}(\gamma) \\
\Sigma\left(\beta^{*}\right)=\Sigma(\beta) & \mathcal{L}\left(\beta^{*}\right)=(\mathcal{L}(\beta))^{*}
\end{array}
$$

where for sets $M$ and $N$ of words, $M . N=\{\alpha \beta \mid \alpha \in M, \beta \in N\}, M^{0}=\{\varepsilon\}(\varepsilon$ denotes the empty word), $M^{n+1}=M \cdot M^{n}$ for $n \geq 0$, and $M^{*}=\bigcup_{n \geq 0} M^{n}$.

We will use letters like $\omega$ to denote either an atomic program from $\Sigma$ or a test (of the form $\varphi$ ?). A word $\omega_{1} \ldots \omega_{k} \in \mathcal{L}(\alpha)$ can be treated as the program $\left(\omega_{1} ; \ldots ; \omega_{k}\right)$, especially when interpreted in a Kripke model.

Recall that a finite automaton $A$ over an alphabet $\Sigma(\alpha)$ is a tuple $\langle\Sigma(\alpha), Q, I, \delta, F\rangle$, where $Q$ is a finite set of states, $I \subseteq Q$ is the set of initial states, $\delta \subseteq Q \times \Sigma(\alpha) \times Q$ is the transition relation, and $F \subseteq Q$ is the set of accepting states. A run of $A$ on a word $\omega_{1} \ldots \omega_{k}$ is a finite sequence of states $q_{0}, q_{1}, \ldots, q_{k}$ such that $q_{0} \in I$ and $\delta\left(q_{i-1}, \omega_{i}, q_{i}\right)$ holds for every $1 \leq i \leq k$. It is an accepting run if $q_{k} \in F$. We say that $A$ accepts a word $w$ if there exists an accepting run of $A$ on $w$. The set of words accepted by $A$ is denoted by $\mathcal{L}(A)$.

We will use the following convention:

\footnotetext{
${ }^{2}$ Note that $\Sigma(\alpha)$ contains not only atomic programs but also expressions of the form $(\varphi$ ?), and a program $\alpha$ is a regular expression over its alphabet $\Sigma(\alpha)$.
} 
- given a finite automaton $A$, we always assume that $A=\left(\Sigma_{A}, Q_{A}, I_{A}, \delta_{A}, F_{A}\right)$,

- for $q \in Q_{A}$, we define $\delta_{A}(q)=\left\{\left(\omega, q^{\prime}\right) \mid\left(q, \omega, q^{\prime}\right) \in \delta_{A}\right\}$.

As a finite automaton $A$ over an alphabet $\Sigma(\alpha)$ corresponds to a program (the regular expression recognizing the same language), it is interpreted in a Kripke model $\mathcal{M}$ as follows:

$$
A^{\mathcal{M}}=\bigcup\left\{\gamma^{\mathcal{M}} \mid \gamma \in \mathcal{L}(A)\right\} .
$$

For each program $\alpha$, let $\mathbb{A}_{\alpha}$ be a finite automaton recognizing the regular language $\mathcal{L}(\alpha)$. The automaton $\mathbb{A}_{\alpha}$ can be constructed from $\alpha$ in polynomial time. We extend the base language with the auxiliary modal operators $[A, q]$ and $\langle A, q\rangle$, where $A$ is $\mathbb{A}_{\alpha}$ for some program $\alpha$ and $q$ is a state of $A$. Here, $[A, q]$ and $\langle A, q\rangle$ stand respectively for $[(A, q)]$ and $\langle(A, q)\rangle$, where $(A, q)$ is the automaton that differs from $A$ only in that $q$ is its only initial state. We call $[A, q]$ (resp. $\langle A, q\rangle)$ a universal (resp. existential) automaton-modal operator.

In the extended language of HPDL, if $\varphi$ is a formula, then $[A, q] \varphi$ and $\langle A, q\rangle \varphi$ are also formulas. The semantics of these formulas are defined as usual, treating $(A, q)$ as a program with semantics specified by (11). From now on, the extended language is used instead of the base language.

Given a Kripke model $\mathcal{M}$ and a state $x \in \Delta^{\mathcal{M}}$, we have $x \in([A, q] \varphi)^{\mathcal{M}}$ (resp. $\left.x \in(\langle A, q\rangle \varphi)^{\mathcal{M}}\right)$ iff

$x_{k} \in \varphi^{\mathcal{M}}$ for all (resp. some) $x_{k} \in \Delta^{\mathcal{M}}$ such that there exist a word $\omega_{1} \ldots \omega_{k}$

(with $k \geq 0$ ) accepted by $(A, q)$ with $\left(x, x_{k}\right) \in\left(\omega_{1} ; \ldots ; \omega_{k}\right)^{\mathcal{M}}$.

The condition $\left(x, x_{k}\right) \in\left(\omega_{1} ; \ldots ; \omega_{k}\right)^{\mathcal{M}}$ means there exist states $x_{0}=x, x_{1}, \ldots, x_{k-1}$ of $\mathcal{M}$ such that, for each $1 \leq i \leq k$, if $\omega_{i} \in \Sigma$ then $\left(x_{i-1}, x_{i}\right) \in \omega_{i}^{\mathcal{M}}$, else $\omega_{i}=\left(\psi_{i}\right.$ ? $)$ for some $\psi_{i}$ and $x_{i-1}=x_{i}$ and $x_{i} \in \psi_{i}^{\mathcal{M}}$. Clearly, $\langle A, q\rangle$ is dual to $[A, q]$ in the sense that $\langle A, q\rangle \varphi \equiv \neg[A, q] \neg \varphi$ for any formula $\varphi$.

\section{A Tableau Calculus for HPDL}

From now on, let $\Gamma$ be an ABox in NNF. In this section, we present a tableau calculus $\mathcal{C}_{\text {HPDL }}$ for checking whether $\Gamma$ is satisfiable. We specify the data structure, the tableau rules, the corresponding tableau decision procedure and state its properties.

\subsection{The Data Structure}

Let EdgeLabels be the set of formulas and assertions of the form $\langle\sigma\rangle \varphi$ or $a:\langle\sigma\rangle \varphi$.

Definition 3.1. A tableau is a rooted graph $G=(V, E, \nu)$, where $V$ is a set of nodes, $E \subseteq V \times V$ is a set of edges, $\nu \in V$ is the root, each node $v \in V$ has a number of attributes, and each edge $(v, w)$ may be labeled by a set ELabels $(v, w) \subseteq$ EdgeLabels. The attributes of a tableau node $v$ are:

- Type $(v) \in\{$ state, non-state $\}$,

- SType $(v) \in\{$ complex, simple $\}$, called the subtype of $v$,

- Label $(v)$, which is a finite set of assertions or formulas, called the label of $v$,

- Reduced $(v)$, which is a finite set of so called reduced assertions or formulas of $v$,

- Status $(v) \in\{$ unexpanded, expanded, incomplete, blocked, closed $\} \cup\{$ closed-wrt $(U)$ $\mid U \subseteq V$ and, for all $u \in U$, Type $(u)=$ state and SType $(u)=$ complex $\}$, 
- AssSugByNom $(v)$, which is a finite set of so called assertions suggested by nominals for $v$, available (i.e., $\neq$ null) only when $\operatorname{SType}(v)=$ complex and Type $(v)=$ state, and is non-empty only when $\operatorname{Status}(v)=$ incomplete,

$-\operatorname{NomRepl}(v): \mathcal{O} \rightarrow \mathcal{O}$, which is a partial mapping specifying replacements of nominals for $v$, called in short the nominal replacement for $v$, and is available (i.e., $\neq$ null) only when SType $(v)=$ complex.

We define

- FullLabel $(v)=\operatorname{Label}(v) \cup \operatorname{Reduced}(v)$ if SType $(v)=$ simple,

$-\operatorname{FullLabel}(v)=\operatorname{Label}(v) \cup \operatorname{Reduced}(v) \cup\{a: b \mid \operatorname{NomRepl}(v)(b)=a\}$ otherwise.

We call $v$ a state if Type $(v)=$ state, and a non-state otherwise. A state is like an "and"-node and a non-state is like an "or"-node, when treating a tableau as an "and-or" graph.

A node $v$ is called a complex node if SType $(v)=$ complex, and a simple node otherwise. The label of a complex node consists of assertions, while the label of a simple node consists of formulas. Using terminology of description logic, a complex node is like an ABox consisting of assertions about named individuals, while a simple node is like an unnamed individual and its label consists of properties of that individual. The root $\nu$ is a complex non-state with $\operatorname{Label}(\nu)=\Gamma$. The assertions/formulas in the label of a node $v$ are treated as requirements to be realized for $v$. Realizing such requirements causes the graph to be expanded or modified.

For the intuition behind Reduced $(v)$, consider an example situation when $\varphi \wedge \psi \in$ $\operatorname{Label}(v)$. To realize the requirement $\varphi \wedge \psi$ for $v$, we can connect $v$ to a node $w$ that differs from $v$ in that $\operatorname{Label}(w)$ contains $\varphi$ and $\psi$ instead of $\varphi \wedge \psi$ and Reduced $(w)=$ $\operatorname{Reduced}(v) \cup\{\varphi \wedge \psi\}$. In general, Reduced $(v)$ contains assertions or formulas that have been reduced for $v$.

$\operatorname{Status}(v)$ is called the status of $v$. Possible statuses of nodes are: unexpanded, expanded, incomplete, closed, blocked and closed-wrt $(U)$, where $U$ is a set of complex states and closed-wrt $(U)$ is read as "closed w.r.t. any node from $U$ ". A node $v$ may have status incomplete only when it is a complex state, and this status means that we would like to extend the label of $v$ with the assertions from $A s s S u g B y N o m(v)$ as one of the possibilities. Informally, closed means "unsatisfiable" and closed-wrt $(U)$ means "unsatisfiable w.r.t. any node from $U$ ". By closed-wrt(...) we denote closed-wrt $(U)$ for some $U$, and by closed-wrt $t_{+}(u)$ we denote closed-wrt $(U)$ for some $U$ containing $u$. When negated, e.g., in the form $\neq$ closed-wrt ${ }_{+}(u)$ or $\notin\left\{\right.$ closed-wrt $\left._{+}(u), \ldots\right\}$, we mean the considered status is different from $\operatorname{closed-wrt}(U)$ for any $U$ that contains $u$. A node may have status blocked only when it is a simple node whose label contains some nominals. The status blocked can be updated only to closed or closed-wrt(...).

A fact $\operatorname{NomRepl}(v)(b)=a$ means that the nominal $b$ has been replaced by $a$ for the complex node $v$. For example, this can be due to an assertion $a: b$.

An edge departing from a node $v$ is labeled if and only if $v$ is a state. If $(v, w) \in E$, then we call $v$ a predecessor of $w$ and $w$ a successor of $v$. Let the relation "being an ancestor" be the reflexive-transitive closure of the relation "being a predecessor". We say that $v$ is a descendant of $u$ if $u$ is an ancestor of $v$.

A tableau is constructed with global caching in the sense that, if $v_{1}$ and $v_{2}$ are different nodes, then Type $\left(v_{1}\right) \neq$ Type $\left(v_{2}\right)$ or SType $\left(v_{1}\right) \neq \operatorname{SType}\left(v_{2}\right)$ or Label $\left(v_{1}\right) \neq$ $\operatorname{Label}\left(v_{2}\right)$ or $\operatorname{Reduced}\left(v_{1}\right) \neq \operatorname{Reduced}\left(v_{2}\right)$ or $\operatorname{NomRepl}\left(v_{1}\right) \neq \operatorname{NomRepl}\left(v_{2}\right)$. 

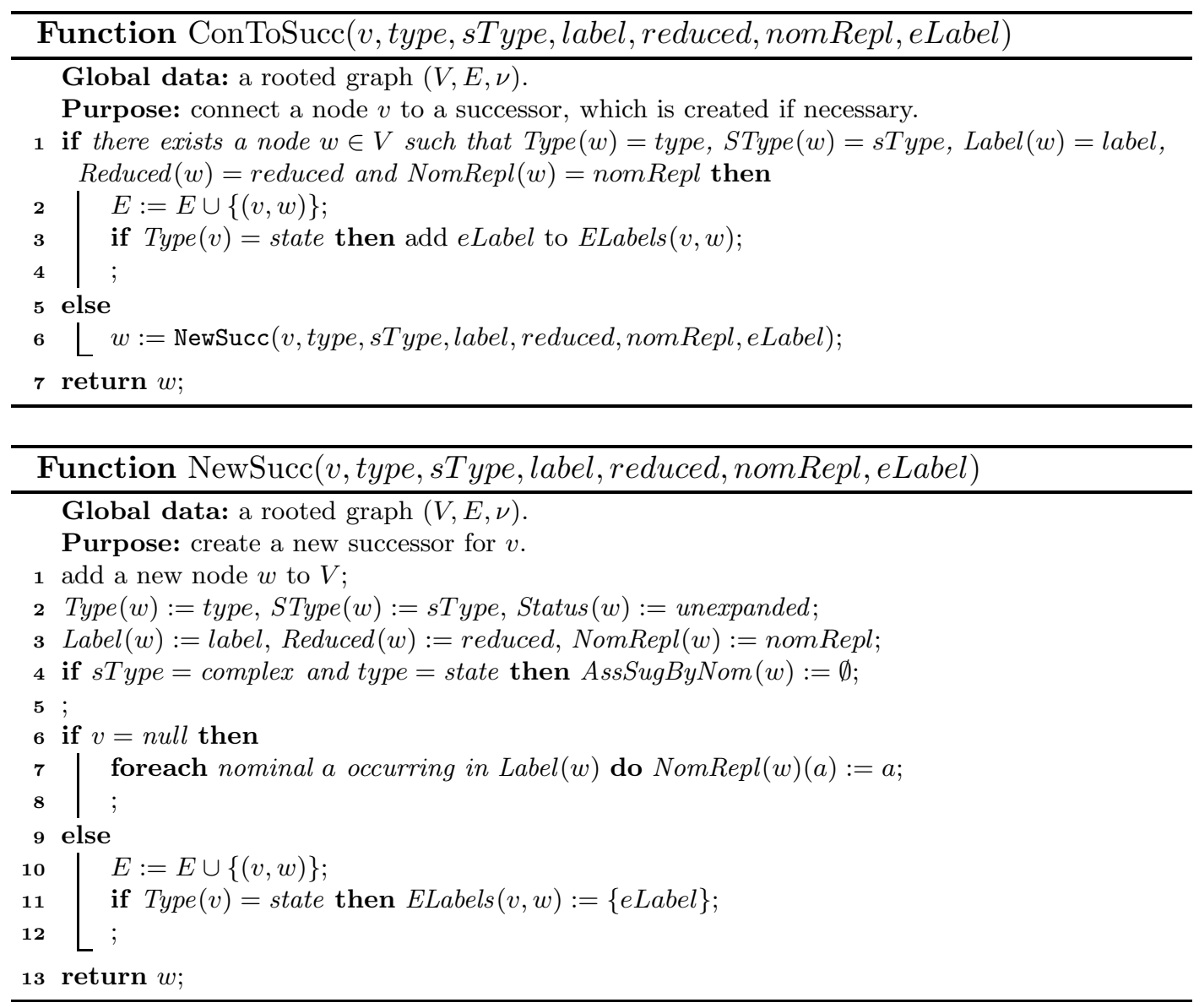

Connecting a node $v$ to a successor, which is created if necessary, is done by Function ConToSucc ( $v$, type, sType, label, reduced, nomRepl, eLabel) on page 7 , where the parameters type, sType, label, reduced, nomRepl specify the attributes of the successor, and eLabel stands for an edge label of the connection. By applying global caching, we first check whether an existing node can be used as such a successor of $v$. If not, a new node is created and used as a successor of $v$ by calling Function NewSucc with the same parameters.

\subsection{Tableau Rules}

Our tableau calculus $\mathcal{C}_{\text {HPDL }}$ consists of the following tableau rules:

- the static rules for expanding a non-state,

- the rule (repl-nom) for replacing nominals in a complex non-state,

- the rule (nominal) for dealing with nominals in a simple non-state,

- the rule (re-expand) for re-expanding a complex non-state,

- the rule (form-state) for forming a state,

- the transitional rule (trans) for expanding a state,

- the rule (close) for updating the status of a node to closed or closed-wrt(...).

The applicability of a rule to a tableau is explicitly specified for the static rules. For any of the other rules, we say that it is applicable to a tableau if its execution can make changes to the tableau. 


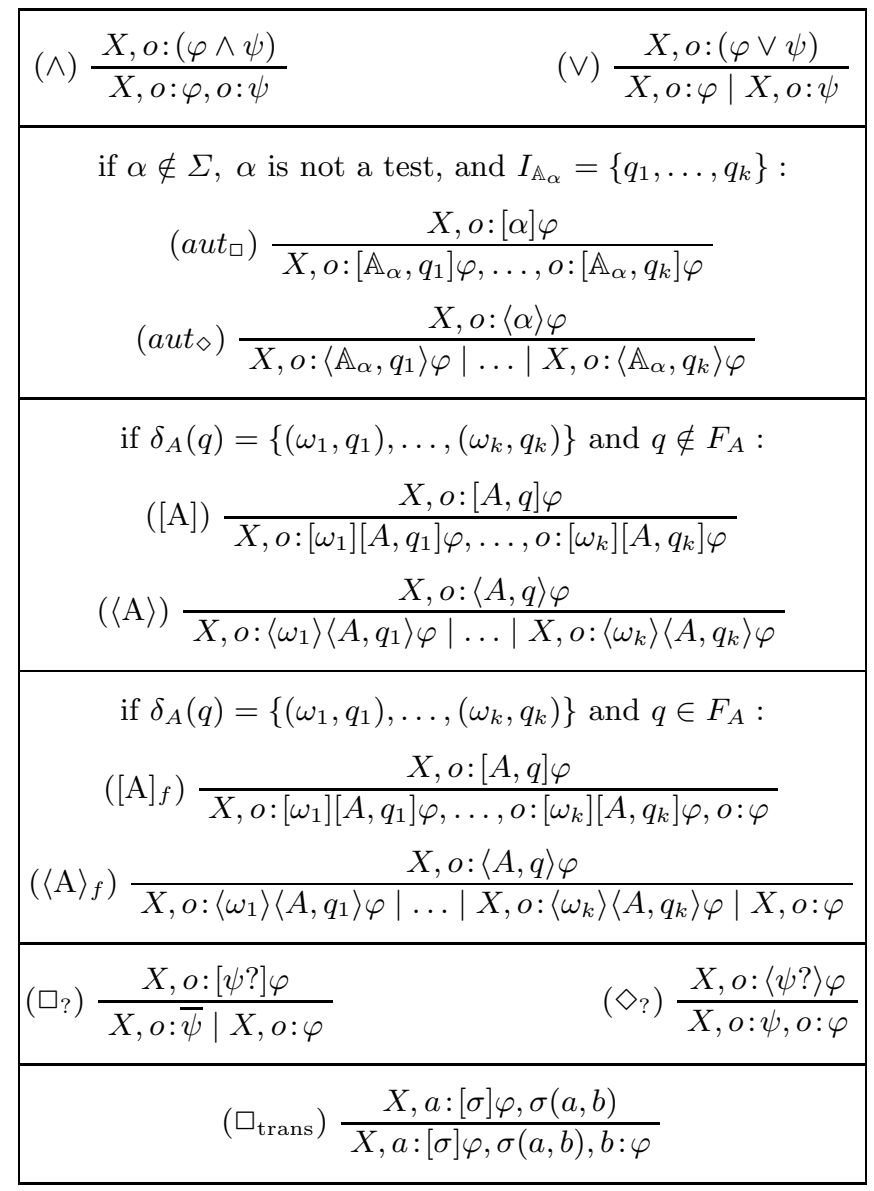

Table 1. The static rules of $\mathcal{C}_{\mathrm{HPDL}}$.

\section{The static rules for expanding a non-state:}

The static rules are written downwards, with a set of assertions/formulas above the line as the premise, which represents the label of the node to which the rule is applied, and a number of sets of assertions/formulas below the line as the (possible) conclusions, which represent the labels of the successor nodes resulted from the application of the rule. Possible conclusions of a static rule are separated by $\mid$. If a rule is unary (i.e., with only one possible conclusion), then its only conclusion is "firm" and we ignore the word "possible". The meaning of a static rule is that, if the premise is satisfiable, then some of the possible conclusions are also satisfiable.

We use $X$ and $Y$ to denote sets of assertions/formulas and write $X, o: \varphi$ to denote $X \cup\{o: \varphi\}$ with the assumption that $o: \varphi \notin X$. The static rules of $\mathcal{C}_{\text {HPDL }}$ are specified in Table 1 as schemas. For each of them, the distinguished assertions/formulas of the premise are called the principal assertions/formulas of the rule. A static rule $(\rho)$ as an instance of a schema given in Table 1 is applicable to a node $v$ if the following conditions hold:

- Status $(v)=$ unexpanded and Type $(v)=$ non-state,

- the rules (repl-nom) and (nominal) are not applicable to $v$,

- the premise of the rule is equal to $\operatorname{Label}(v)$,

- the conditions accompanied with $(\rho)$ are satisfied, 
- if $(\rho) \neq\left(\square_{\text {trans }}\right)$, then the principal assertion/formula of $(\rho)$ does not belong to $\operatorname{Reduced}(v)$, else the assertion $b: \varphi$ in the conclusion does not belong to FullLabel $(v)$.

The last condition prevents applying the rule unnecessarily, because it has been applied to an ancestor node of $v$ that corresponds to the same state in the intended Kripke model.

If $(\rho) \neq\left(\square_{\text {trans }}\right)$ is a static rule applicable to $v$, then the application is as follows:

- Let $\xi$ be the principal assertion/formula of $(\rho)$.

- Let $X_{1}, \ldots, X_{k}$ be the possible conclusions of $(\rho)$.

- For each $1 \leq i \leq k$, do $\operatorname{ConTo\operatorname {Succ}}\left(v\right.$, non-state, $\operatorname{SType}(v), X_{i}, \operatorname{Reduced}(v) \cup\{\xi\}$, $\operatorname{NomRepl}(v)$, null), which is specified on page $7^{3}$

$-\operatorname{Status}(v):=$ expanded.

If ( $\square_{\text {trans }}$ ) is applicable to $v$, then the application is as follows:

- Let $Y$ be the conclusion of ( $\left.\square_{\text {trans }}\right)$.

- ConToSucc ( $v$, non-state, SType $(v), Y, \operatorname{Reduced}(v), \operatorname{NomRepl}(v)$, null $)$.

$-\operatorname{Status}(v):=$ expanded.

Applying a static rule understood as a schema to a node $v$ means applying an instance of the schema to $v$. Such an instance is chosen as follows: choose assertions/formulas from $\operatorname{Label}(v)$ such that they can be "unified" with the principal assertions/formulas in the schema, then instantiate the schema by using the substitution resulted from that unification.

\section{The rule (repl-nom) for replacing nominals:}

If $\operatorname{Status}(v)=$ unexpanded and $\operatorname{Label}(v)$ contains $a: b$ with $a \neq b$ then:

1. let $X$ and $Y$ be the sets obtained from $\operatorname{Label}(v)-\{a: b\}$ and Reduced $(v)$, respectively, by replacing every occurrence of $b$ with $a$, including the ones in automata of modal operators;

2. $w:=\operatorname{ConToSucc}(v$, non-state, complex, $X, Y, \operatorname{NomRepl}(v)$, null $)$;

3. $\operatorname{NomRepl}(w)(b):=a$;

4. for each nominal $c$ such that $\operatorname{NomRepl}(v)(c)=b$, do $\operatorname{NomRepl}(w)(c):=a$;

5. $\operatorname{Status}(v):=$ expanded;

\section{The rule (nominal) for dealing with nominals:}

If $\operatorname{Status}(v) \neq$ closed, $\operatorname{Type}(v)=\operatorname{simple}$ and there exists $a \in \operatorname{Label}(v)$, then:

1. for each complex state $u$ such that $\operatorname{Status}(v) \neq \operatorname{closed}_{\text {-wrt }}(u)$ and $\operatorname{Status}(u) \neq$ incomplete and $v$ may affect the status of the root $\nu$ via a path through $u, 4$ do:

(a) $X:=\{a: \varphi \mid \varphi \in \operatorname{Label}(v)$ and $\varphi \neq a\}$;

(b) if there exists $\xi \in X$ such that $\bar{\xi} \in \operatorname{FullLabel}(u)$ then

i. if $\operatorname{Status}(v)$ is of the form closed-wrt $(U)$ then $\operatorname{Status}(v):=$ closed-wrt $(U \cup\{u\})$,

\footnotetext{
${ }^{3}$ Here, null is a constant standing for "nothing" as in C programming, which means the parameter is not important for this case.

${ }^{4}$ That is, there exists a path from $\nu$ to $v$ via $u$ that does not contain any node with status closed or closed-wrt $+(u)$.
} 
ii. else $\operatorname{Status}(v):=$ closed-wrt $(\{u\})$;

(c) else if $X \nsubseteq$ FullLabel $(u)$ then

i. $\operatorname{Status}(u):=$ incomplete;

ii. $\operatorname{AssSugByNom}(u):=X-\operatorname{FullLabel}(u)$;

2. if $\operatorname{Status}(v)=$ unexpanded then Status $(v):=$ blocked.

The rule (re-expand) for re-expanding a complex non-state:

If $(v, w) \in E$ and Status $(w)=$ incomplete then:

(we must have that $\operatorname{SType}(v)=$ complex and Type $(v)=$ non-state)

1. delete the edge $(v, w)$ from $E$;

2. $X:=\operatorname{Label}(v) \cup$ AssSugByNom $(w)$;

3. ConToSucc ( $v$, non-state, SType (v), X, Reduced ( $v), \operatorname{NomRepl}(v)$, null);

4. for each $\xi \in A s s \operatorname{SugByNom}(w)$, do $\operatorname{ConToSucc}(v$, non-state, SType $(v)$, Label $(v) \cup$ $\{\bar{\xi}\}, \operatorname{Reduced}(v), \operatorname{NomRepl}(v)$, null $)$.

The rule (form-state) for forming a state:

If Status $(v)=$ unexpanded, Type $(v)=$ non-state and no rule among the static rules, (repl-nom) and (nominal) is applicable to $v$, then:

1. if SType $(v)=$ complex then

ConToSucc $(v$, state, complex, Label $(v), \operatorname{Reduced}(v), \operatorname{NomRepl}(v), n u l l)$,

2. else ConToSucc ( $v$, state, simple, Label $(v), \emptyset$, null, null);

3. $\operatorname{Status}(v):=$ expanded.

We need the set Reduced $(v)$ for the successor of $v$ in the case SType $(v)=$ complex due to the situation related with the rule (nominal).

\section{The transitional rule (trans) for expanding a state:}

If Type $(v)=$ state and Status $(v)=$ unexpanded then:

1. for each $o:\langle\sigma\rangle \varphi \in \operatorname{Label}(v)$ do

(a) $X:=\{\varphi\} \cup\{\psi \mid o:[\sigma] \psi \in \operatorname{Label}(v)\}$;

(b) $\operatorname{ConToSucc}(v$, non-state, simple, $X, \emptyset$, null, $o:\langle\sigma\rangle \varphi)$;

2. $\operatorname{Status}(v):=$ expanded.

The rule (close) for updating the status of a node:

We need the following definition before specifying the rule (close).

Definition 3.2. Let $\xi \in$ FullLabel $(v)$ be of the form $o:\langle A, q\rangle \varphi$ or $o:\langle\omega\rangle\langle A, q\rangle \varphi$. We say that $\xi$ is $\diamond$-realizable at $v$ w.r.t. $u$, where $u$ is a complex state and an ancestor of $v$ such that $\operatorname{Status}(u) \notin\{$ closed, incomplete $\}$, iff the following conditions hold:

1. Status $(v) \notin\left\{\right.$ closed, closed-wrt $\left.{ }_{+}(u)\right\}$,

2. either $\operatorname{Status}(v) \in\{$ unexpanded, incomplete $\}$ or some of the following conditions hold:

(a) $\xi=o:\langle A, q\rangle \varphi, q \in F_{A}$ and $o: \varphi \in$ FullLabel $(v)$; 
(b) $\xi=o:\langle A, q\rangle \varphi,\left(q, \omega, q^{\prime}\right) \in \delta_{A}, o:\langle\omega\rangle\left\langle A, q^{\prime}\right\rangle \varphi$ belongs to FullLabel $(v)$ and is $\diamond$-realizable at $v$ w.r.t. $u$;

(c) $\xi=o:\langle\psi ?\rangle\langle A, q\rangle \varphi,\{o: \psi, o:\langle A, q\rangle \varphi\} \subseteq$ FullLabel $(v)$ and $o:\langle A, q\rangle \varphi$ is $\diamond$ realizable at $v$ w.r.t. $u$;

(d) $v$ is expanded by the tableau rule $\left(\langle\mathrm{A}\rangle_{f}\right), \xi=o:\langle A, q\rangle \varphi$ is the principal assertion/formula, and the successor $w$ of $v$ whose label is obtained from Label $(v)$ by replacing $\xi$ with $o: \varphi$ has Status $(w) \notin\left\{\right.$ closed, closed-wrt - $\left._{+}(u)\right\}$;

(e) $v$ is expanded by the tableau rule $(\langle\mathrm{A}\rangle)$ or $\left(\langle\mathrm{A}\rangle_{f}\right), \xi=o:\langle A, q\rangle \varphi$ is the principal assertion/formula, $w$ is a successor of $v$ and the assertion/formula $\xi^{\prime} \in \operatorname{Label}(w)$ obtained from $\xi$ is $\diamond$-realizable at $w$ w.r.t. $u$;

(f) $v$ is expanded by the tableau rule $\left(\diamond_{?}\right), \xi=o:\langle\psi ?\rangle\langle A, q\rangle \varphi$ is the principal assertion/formula, $w$ is the unique successor of $v$, and $o:\langle A, q\rangle \varphi$ is $\diamond$-realizable at $w$ w.r.t. $u$;

(g) $v$ is expanded by the rule (re-expand), (form-state) or a static tableau rule and $\xi$ is not a principal assertion/formula, and $\xi$ is $\diamond$-realizable at a successor of $v$ w.r.t. $u$;

(h) $v$ is expanded by the rule (trans), $\xi=o:\langle\sigma\rangle\langle A, q\rangle \varphi, w$ is a successor of $v, \xi \in$ ELabels $(v, w),\langle A, q\rangle \varphi \in \operatorname{Label}(w)$, and $\langle A, q\rangle \varphi$ is $\diamond$-realizable at $w$ w.r.t. $u$;

(i) there exists $a \in \operatorname{Label}(v)$ such that $a: \xi$ is $\diamond$-realizable at $u$ w.r.t. $u$.

Observe that the notion of $\diamond$-realizability is defined inductively and the conditions $2 \mathrm{a}$ and $2 \mathrm{~d}$ correspond to the base cases.

The rule (close) is specified as follows:

1. If $\operatorname{Status}(v) \neq$ closed then:

(a) if (there exists $o: \perp \in \operatorname{Label}(v)$ or $a: \neg a \in \operatorname{Label}(v)$ or $\{\xi, \bar{\xi}\} \subseteq$ FullLabel $(v)$ ) or $\operatorname{Status}(v)=$ closed-wrt $(v)$, then $\operatorname{Status}(v):=$ closed 5

(b) else if $u$ is a complex state and an ancestor of $v$ such that $\operatorname{Status}(u) \notin$ $\{$ closed, incomplete $\}$ and there exists $\xi \in$ FullLabel $(v)$ of the form $o:\langle A, q\rangle \varphi$ or $o:\langle\omega\rangle\langle A, q\rangle \varphi$ that is not $\diamond$-realizable at $v$ w.r.t. $u$ and $\operatorname{Status}(v) \neq$ closed-wrt ${ }_{+}(u)$, then:

i. if $\operatorname{Status}(v)$ is of the form closed-wrt $(U)$, then $\operatorname{Status}(v):=\operatorname{closed}-w r t(U \cup\{u\})$,

ii. else $\operatorname{Status}(v):=$ closed-wrt $(\{u\})$.

2. If $\operatorname{Status}(v) \notin\{$ unexpanded, closed,blocked, closed-wrt $(\ldots)\}$ and Type $(v)=$ non-state, then:

(a) if all successors of $v$ have status closed then $\operatorname{Status}(v):=$ closed,

(b) else if every successor of $v$ has status closed or closed-wrt(...) then:

i. let $w_{1}, \ldots, w_{k}$ be all the successors of $v$ such that, for $1 \leq i \leq k, \operatorname{Status}\left(w_{i}\right)$ is of the form closed-wrt $\left(U_{i}\right)$, and let $U=\bigcap_{1 \leq i \leq k} U_{i}$;

ii. if $U \neq \emptyset$ then: if $\operatorname{Status}(v)$ is of the form $\operatorname{close} \bar{d}-\operatorname{wrt}\left(U^{\prime}\right)$, then $\operatorname{Status}(v):=$ closed-wrt $\left(U^{\prime} \cup U\right)$, else $\operatorname{Status}(v):=$ closed-wrt $(U)$.

3. If Status $(v) \notin\{$ unexpanded, closed, incomplete $\}$ and Type $(v)=$ state, then:

\footnotetext{
${ }^{5}$ As an optimization, if there exists $\xi \in$ FullLabel $(v)$ of the form $o:\langle A, q\rangle \varphi$ or $o:\langle\omega\rangle\langle A, q\rangle \varphi$ such that it is not $\diamond$-realizable at $v$ w.r.t. a complex state $u$ that is an ancestor of $v$ and checking $\diamond$-realizability of $\xi$ at $v$ w.r.t. $u$ does not go through any simple non-state whose label contains a nominal, then $\diamond$-nonrealizability of $\xi$ at $v$ does not really depend on $u$, and $\operatorname{Status}(v)$ can be changed to closed.
} 
(a) if $v$ has a successor $w$ with $\operatorname{Status}(w)=\operatorname{closed}$, then $\operatorname{Status}(v):=$ closed,

(b) else if $v$ has a successor $w$ with $\operatorname{Status}(w)=\operatorname{closed-wrt}(U)$ and $\operatorname{Status}(v)$ is not of the form closed-wrt $\left(U^{\prime}\right)$ with $U^{\prime} \supseteq U$, then:

i. if $\operatorname{Status}(v)$ is of the form closed-wrt $\left(U^{\prime}\right)$, then $\operatorname{Status}(v):=$ closed-wrt $\left(U^{\prime} \cup U\right)$,

ii. else $\operatorname{Status}(v):=$ closed-wrt $(U)$.

\subsection{Checking Satisfiability}

Let $\Gamma$ be an ABox in NNF. A $\mathcal{C}_{\mathrm{HPDL}}$-tableau for $\Gamma$ is a tableau $G=(V, E, \nu)$ constructed as follows. At the beginning, $V:=\emptyset, E:=\emptyset$ and $\nu:=\operatorname{NewSucc}($ null, non-state, complex, $\Gamma, \emptyset, \emptyset$, null). Then, while Status $(\nu) \neq$ closed and there is some tableau rule $(\rho)$ applicable to some node $v$, choose such a pair $((\rho), v)$ and apply $(\rho)$ to $v 6$ Observe that the set of all assertions and formulas that may appear in the contents of the nodes of $G$ is finite. Due to global caching, $G$ is finite and can be effectively constructed. The following theorem immediately follows from Corollaries 5.8 and 5.17, which are given and proved in Section 5.

Theorem 3.3 (Soundness and Completeness). Let $\Gamma$ be an ABox in NNF and $G=(V, E, \nu)$ an arbitrary $\mathcal{C}_{\mathrm{HPDL}}$-tableau for $\Gamma$. Then, $\Gamma$ is satisfiable if and only if Status $(\nu) \neq$ closed.

To check satisfiability of an ABox $\Gamma$ in NNF, one can construct a $\mathcal{C}_{\mathrm{HPDL}}$-tableau $G=(V, E, \nu)$ for $\Gamma$ and return "no" when $\operatorname{Status}(\nu)=$ closed, or "yes" otherwise. We call this the $\mathcal{C}_{\mathrm{HPDL}}$-tableau decision procedure. The following corollary immediately follows from Corollary 5.4, which is given and proved in Section 5 .

Corollary 3.4. The $\mathcal{C}_{\mathrm{HPDL}}$-tableau decision procedure has the EXPTIME complexity.

\section{An Illustrative Example}

Consider the following ABox in NNF:

$$
\Gamma=\left\{a:\left[\sigma^{*}\right] p, \sigma(a, b), b:\left\langle(a ? \cup \sigma)^{*}\right\rangle \neg p\right\} .
$$

We show that $\Gamma$ is unsatisfiable by constructing a $\mathcal{C}_{\mathrm{HPDL}}$-tableau $G=(V, E, \nu)$ for $\Gamma$ with $\operatorname{Status}(\nu)=$ closed. In the construction, we use the following finite automata:

$$
\begin{aligned}
& A_{1}=\mathbb{A}_{\sigma^{*}}=(\{\sigma\},\{0\},\{0\},\{(0, \sigma, 0)\},\{0\}), \\
& A_{2}=\mathbb{A}_{(a ? \cup \sigma)^{*}}=(\{\sigma, a ?\},\{0\},\{0\},\{(0, \sigma, 0),(0, a ?, 0)\},\{0\}), \\
& A_{3}=\mathbb{A}_{(b ? \cup \sigma)^{*}}=(\{\sigma, b ?\},\{0\},\{0\},\{(0, \sigma, 0),(0, b ?, 0)\},\{0\}) .
\end{aligned}
$$

As these automata have only one state (named 0$)$, we will write $A_{i}$ instead of $\left(A_{i}, 0\right)$, for $1 \leq i \leq 3$. For example, $\left[A_{1}\right]$ and $\left\langle A_{1}\right\rangle$ stand for $\left[A_{1}, 0\right]$ and $\left\langle A_{1}, 0\right\rangle$, respectively. The constructed $\mathcal{C}_{\text {HPDL }}$-tableau $G$ is illustrated in Figure 2 ,

At the beginning, $G$ contains the root $\nu$ with $\operatorname{Label}(\nu)=\Gamma$.

\footnotetext{
${ }^{6}$ As an optimization, it makes sense to expand $v$ only when there may exist a path from the root to $v$ that does not contain any node with the status closed.
} 


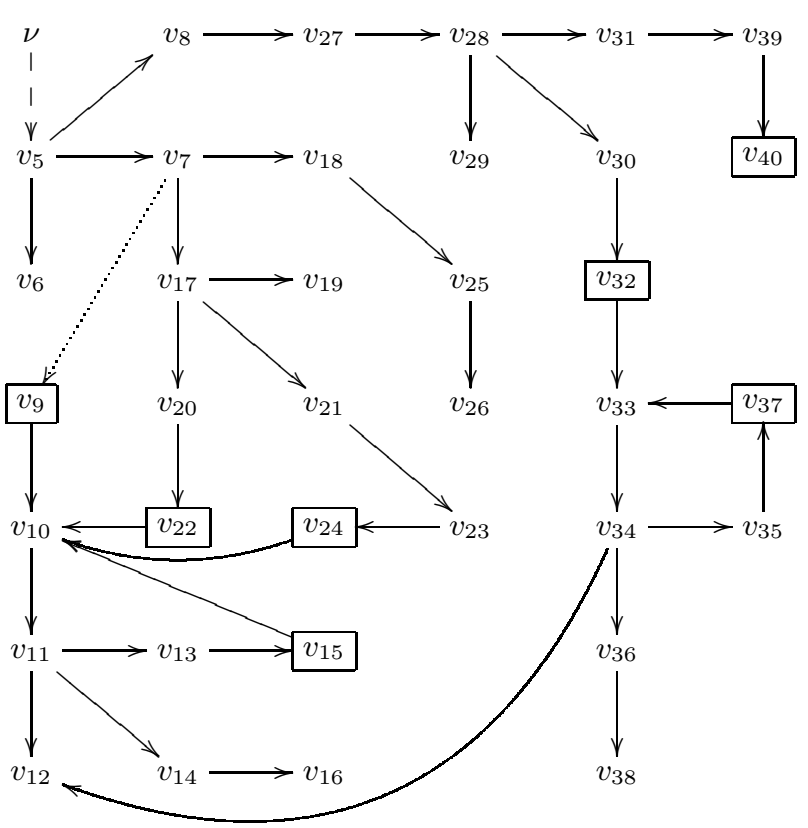

Fig. 2. An illustration for Section 4. The dashed edge from $\nu$ to $v_{5}$ represents a path $\nu \rightarrow v_{1} \rightarrow \ldots \rightarrow v_{5}$. The dotted edge from $v_{7}$ to $v_{9}$ stands for a normal edge before re-expanding $v_{7}$. By the re-expansion, that edge is deleted and $v_{7}$ is connected to the newly created nodes $v_{17}$ and $v_{18}$. The nodes in rectangular frames are states, the others are non-states. The nodes $v_{10}-v_{16}$ and $v_{33}-v_{38}$ are simple nodes, the others are complex nodes. The nodes $v_{16}$ and $v_{38}$ have the status blocked.

Applying $\left(a u t_{\square}\right), \nu$ is connected to a new complex non-state $v_{1}$ with

$$
\operatorname{Label}\left(v_{1}\right)=\left\{a:\left[A_{1}\right] p, \sigma(a, b), b:\left\langle(a ? \cup \sigma)^{*}\right\rangle \neg p\right\} .
$$

Applying $\left([\mathrm{A}]_{f}\right), v_{1}$ is connected to a new complex non-state $v_{2}$ with

$$
\operatorname{Label}\left(v_{2}\right)=\left\{a:[\sigma]\left[A_{1}\right] p, a: p, \sigma(a, b), b:\left\langle(a ? \cup \sigma)^{*}\right\rangle \neg p\right\} .
$$

Applying ( $\left.\square_{\text {trans }}\right), v_{2}$ is connected to a new complex non-state $v_{3}$ with

$$
\operatorname{Label}\left(v_{3}\right)=\operatorname{Label}\left(v_{2}\right) \cup\left\{b:\left[A_{1}\right] p\right\} .
$$

Applying $\left([\mathrm{A}]_{f}\right), v_{3}$ is connected to a new complex non-state $v_{4}$ with

$$
\operatorname{Label}\left(v_{4}\right)=\operatorname{Label}\left(v_{2}\right) \cup\left\{b:[\sigma]\left[A_{1}\right] p, b: p\right\} .
$$

Applying $\left(a u t_{\diamond}\right), v_{4}$ is connected to a new complex non-state $v_{5}$ with

$$
\operatorname{Label}\left(v_{5}\right)=\left\{a:[\sigma]\left[A_{1}\right] p, a: p, \sigma(a, b), b:[\sigma]\left[A_{1}\right] p, b: p, b:\left\langle A_{2}\right\rangle \neg p\right\} .
$$

Applying $\left(\langle\mathrm{A}\rangle_{f}\right), v_{5}$ is connected to new complex non-states $v_{6}, v_{7}$, and $v_{8}$ with

$$
\begin{aligned}
& \text { Label }\left(v_{6}\right)=\left\{a:[\sigma]\left[A_{1}\right] p, a: p, \sigma(a, b), b:[\sigma]\left[A_{1}\right] p, b: p, b: \neg p\right\} \\
& \text { Label }\left(v_{7}\right)=\left\{a:[\sigma]\left[A_{1}\right] p, a: p, \sigma(a, b), b:[\sigma]\left[A_{1}\right] p, b: p, b:\langle\sigma\rangle\left\langle A_{2}\right\rangle \neg p\right\}, \\
& \operatorname{Label}\left(v_{8}\right)=\left\{a:[\sigma]\left[A_{1}\right] p, a: p, \sigma(a, b), b:[\sigma]\left[A_{1}\right] p, b: p, b:\langle a ?\rangle\left\langle A_{2}\right\rangle \neg p\right\} .
\end{aligned}
$$


Applying (close), Status $\left(v_{6}\right)$ is changed to closed.

Applying (form-state), $v_{7}$ is connected to a new complex state $v_{9}$ with $\operatorname{Label}\left(v_{9}\right)=$ Label $\left(v_{7}\right)$. Applying (trans), $v_{9}$ is connected to a new simple non-state $v_{10}$ with

$$
\begin{aligned}
\operatorname{Label}\left(v_{10}\right) & =\left\{\left\langle A_{2}\right\rangle \neg p,\left[A_{1}\right] p\right\}, \\
\text { ELabels }\left(v_{9}, v_{10}\right) & =\left\{b:\langle\sigma\rangle\left\langle A_{2}\right\rangle \neg p\right\} .
\end{aligned}
$$

Applying $\left([\mathrm{A}]_{f}\right), v_{10}$ is connected to a new simple non-state $v_{11}$ with

$$
\operatorname{Label}\left(v_{11}\right)=\left\{\left\langle A_{2}\right\rangle \neg p,[\sigma]\left[A_{1}\right] p, p\right\} .
$$

Applying $\left(\langle\mathrm{A}\rangle_{f}\right), v_{11}$ is connected to new simple non-states $v_{12}, v_{13}$, and $v_{14}$ with

$$
\begin{aligned}
& \operatorname{Label}\left(v_{12}\right)=\left\{\neg p,[\sigma]\left[A_{1}\right] p, p\right\}, \\
& \operatorname{Label}\left(v_{13}\right)=\left\{\langle\sigma\rangle\left\langle A_{2}\right\rangle \neg p,[\sigma]\left[A_{1}\right] p, p\right\}, \\
& \operatorname{Label}\left(v_{14}\right)=\left\{\langle a ?\rangle\left\langle A_{2}\right\rangle \neg p,[\sigma]\left[A_{1}\right] p, p\right\} .
\end{aligned}
$$

Applying (close), Status $\left(v_{12}\right)$ is changed to closed.

Applying (form-state), $v_{13}$ is connected to a new simple state $v_{15}$ with $\operatorname{Label}\left(v_{15}\right)=$ Label $\left(v_{13}\right)$. Applying (trans), $v_{15}$ is connected to the existing node $v_{10}$ with ELabels $\left(v_{15}, v_{10}\right)=\left\{\langle\sigma\rangle\left\langle A_{2}\right\rangle \neg p\right\}$.

Applying $\left(\diamond_{?}\right), v_{14}$ is connected to a new simple non-state $v_{16}$ with

$$
\operatorname{Label}\left(v_{16}\right)=\left\{a,\left\langle A_{2}\right\rangle \neg p,[\sigma]\left[A_{1}\right] p, p\right\} .
$$

Applying (nominal) to $v_{16}$ and the complex state $v_{9}$, Status $\left(v_{9}\right)$ is changed to incomplete, AssSugByNom $\left(v_{9}\right)$ is set to $\left\{a:\left\langle A_{2}\right\rangle \neg p\right\}$, and $\operatorname{Status}\left(v_{16}\right)$ is changed to blocked.

Applying (re-expand) to $v_{7}$ and the incomplete complex state $v_{9}$, the edge $\left(v_{7}, v_{9}\right)$ is deleted and $v_{7}$ is connected to new complex non-states $v_{17}$ and $v_{18}$ with

$$
\begin{aligned}
& \operatorname{Label}\left(v_{17}\right)=\operatorname{Label}\left(v_{7}\right) \cup\left\{a:\left\langle A_{2}\right\rangle \neg p\right\} \\
& \operatorname{Label}\left(v_{18}\right)=\operatorname{Label}\left(v_{7}\right) \cup\left\{a:\left[A_{2}\right] p\right\} .
\end{aligned}
$$

Applying $\left(\langle\mathrm{A}\rangle_{f}\right), v_{17}$ is connected to new complex non-states $v_{19}, v_{20}, v_{21}$ with

$$
\begin{aligned}
& \operatorname{Label}\left(v_{19}\right)=\operatorname{Label}\left(v_{7}\right) \cup\{a: \neg p\}, \\
& \operatorname{Label}\left(v_{20}\right)=\operatorname{Label}\left(v_{7}\right) \cup\left\{a:\langle\sigma\rangle\left\langle A_{2}\right\rangle \neg p\right\}, \\
& \operatorname{Label}\left(v_{21}\right)=\operatorname{Label}\left(v_{7}\right) \cup\left\{a:\langle a ?\rangle\left\langle A_{2}\right\rangle \neg p\right\} .
\end{aligned}
$$

Applying (close), Status $\left(v_{19}\right)$ is changed to closed (due to $a: p$ and $a: \neg p$ ).

Applying (form-state), $v_{20}$ is connected to a new complex state $v_{22}$ with Label $\left(v_{22}\right)=\operatorname{Label}\left(v_{20}\right)$. Applying (trans), $v_{22}$ is connected to the existing node $v_{10}$ with ELabels $\left(v_{22}, v_{10}\right)=\left\{a:\langle\sigma\rangle\left\langle A_{2}\right\rangle \neg p, b:\langle\sigma\rangle\left\langle A_{2}\right\rangle \neg p\right\}$.

Observe that the assertion $b:\langle\sigma\rangle\left\langle A_{2}\right\rangle \neg p$ is not $\diamond$-realizable at $v_{22}$ w.r.t. $v_{22}$. Hence, applying (close), Status $\left(v_{22}\right)$ is first changed to closed-wrt $\left(\left\{v_{22}\right\}\right)$ and then to closed, after that $\operatorname{Status}\left(v_{20}\right)$ is also changed to closed.

Applying $\left(\diamond_{\text {? }}\right), v_{21}$ is connected to a new complex non-state $v_{23}$ with

$$
\operatorname{Label}\left(v_{23}\right)=\operatorname{Label}\left(v_{7}\right) \cup\left\{a: a, a:\left\langle A_{2}\right\rangle \neg p\right\} .
$$


Notice that $a:\left\langle A_{2}\right\rangle \neg p \in \operatorname{Reduced}\left(v_{23}\right)$. Applying (form-state), $v_{23}$ is connected to a new complex state $v_{24}$ with $\operatorname{Label}\left(v_{24}\right)=\operatorname{Label}\left(v_{23}\right)$. Applying (trans), $v_{24}$ is connected to the existing node $v_{10}$ with ELabels $\left(v_{24}, v_{10}\right)=\left\{b:\langle\sigma\rangle\left\langle A_{2}\right\rangle \neg p\right\}$.

Observe that the assertion $b:\langle\sigma\rangle\left\langle A_{2}\right\rangle \neg p$ is not $\diamond$-realizable at $v_{24}$ w.r.t. $v_{24}$. Hence, applying (close), Status $\left(v_{24}\right)$ is first changed to closed-wrt $\left(\left\{v_{24}\right\}\right)$ and then to closed, after that the statuses of $v_{23}, v_{21}$ and $v_{17}$ are changed to closed in subsequent steps.

Applying $\left([\mathrm{A}]_{f}\right), v_{18}$ is connected to a new complex non-state $v_{25}$ with

$$
\operatorname{Label}\left(v_{25}\right)=\operatorname{Label}\left(v_{7}\right) \cup\left\{a:[\sigma]\left[A_{2}\right] p, a:[a ?]\left[A_{2}\right] p\right\} .
$$

Applying $\left(\square_{\text {trans }}\right), v_{25}$ is connected to new complex non-states $v_{26}$ with

$$
\operatorname{Label}\left(v_{26}\right)=\operatorname{Label}\left(v_{25}\right) \cup\left\{b:\left[A_{2}\right] p\right\} .
$$

Applying (close), Status $\left(v_{26}\right)$ is changed to closed (due to $b:\left\langle A_{2}\right\rangle \neg p$ and $b:\left[A_{2}\right] p$ ), and then the statuses of $v_{25}, v_{18}$ and $v_{7}$ are changed to closed in subsequent steps.

Applying $\left(\diamond_{\text {? }}\right), v_{8}$ is connected to a new complex non-state $v_{27}$ with

$$
\operatorname{Label}\left(v_{27}\right)=\left\{a:[\sigma]\left[A_{1}\right] p, a: p, \sigma(a, b), b:[\sigma]\left[A_{1}\right] p, b: p, b: a, b:\left\langle A_{2}\right\rangle \neg p\right\} .
$$

Applying (repl-nom), $v_{27}$ is connected to a new complex non-state $v_{28}$ with

$$
\operatorname{Label}\left(v_{28}\right)=\left\{b:[\sigma]\left[A_{1}\right] p, b: p, \sigma(b, b), b:\left\langle A_{3}\right\rangle \neg p\right\} .
$$

Applying $\left(\langle\mathrm{A}\rangle_{f}\right), v_{28}$ is connected to new complex non-states $v_{29}, v_{30}, v_{31}$ with

$$
\begin{aligned}
& \operatorname{Label}\left(v_{29}\right)=\left\{b:[\sigma]\left[A_{1}\right] p, b: p, \sigma(b, b), b: \neg p\right\}, \\
& \operatorname{Label}\left(v_{30}\right)=\left\{b:[\sigma]\left[A_{1}\right] p, b: p, \sigma(b, b), b:\langle\sigma\rangle\left\langle A_{3}\right\rangle \neg p\right\}, \\
& \operatorname{Label}\left(v_{31}\right)=\left\{b:[\sigma]\left[A_{1}\right] p, b: p, \sigma(b, b), b:\langle b ?\rangle\left\langle A_{3}\right\rangle \neg p\right\} .
\end{aligned}
$$

Applying (close), Status $\left(v_{29}\right)$ is changed to closed.

Notice that $b:\left[A_{1}\right] p \in \operatorname{Reduced}\left(v_{30}\right)$. Applying (form-state), $v_{30}$ is connected to a new complex state $v_{32}$ with $\operatorname{Label}\left(v_{32}\right)=\operatorname{Label}\left(v_{30}\right)$. Applying (trans), $v_{32}$ is connected to a new simple non-state $v_{33}$ with

$$
\begin{aligned}
\operatorname{Label}\left(v_{33}\right) & =\left\{\left\langle A_{3}\right\rangle \neg p,\left[A_{1}\right] p\right\}, \\
\text { ELabels }\left(v_{32}, v_{33}\right) & =\left\{b:\langle\sigma\rangle\left\langle A_{3}\right\rangle \neg p\right\} .
\end{aligned}
$$

Applying $\left([\mathrm{A}]_{f}\right), v_{33}$ is connected to a new simple non-state $v_{34}$ with

$$
\operatorname{Label}\left(v_{34}\right)=\left\{\left\langle A_{3}\right\rangle \neg p,[\sigma]\left[A_{1}\right] p, p\right\} .
$$

Applying $\left(\langle\mathrm{A}\rangle_{f}\right), v_{34}$ is connected to the existing node $v_{12}$ and new simple nonstates $v_{35}$ and $v_{36}$ with

$$
\begin{aligned}
& \operatorname{Label}\left(v_{35}\right)=\left\{\langle\sigma\rangle\left\langle A_{3}\right\rangle \neg p,[\sigma]\left[A_{1}\right] p, p\right\}, \\
& \operatorname{Label}\left(v_{36}\right)=\left\{\langle b ?\rangle\left\langle A_{3}\right\rangle \neg p,[\sigma]\left[A_{1}\right] p, p\right\} .
\end{aligned}
$$

Applying (form-state), $v_{35}$ is connected to a new simple state $v_{37}$ with $\operatorname{Label}\left(v_{37}\right)=$ Label $\left(v_{35}\right)$. Applying (trans), $v_{37}$ is connected to the existing node $v_{33}$ with ELabels $\left(v_{37}, v_{33}\right)=\left\{\langle\sigma\rangle\left\langle A_{3}\right\rangle \neg p\right\}$. 
Applying $\left(\diamond_{?}\right), v_{36}$ is connected to a new simple non-state $v_{38}$ with

$$
\operatorname{Label}\left(v_{38}\right)=\left\{b,\left\langle A_{3}\right\rangle \neg p,[\sigma]\left[A_{1}\right] p, p\right\} .
$$

Applying (nominal), Status $\left(v_{38}\right)$ is changed to blocked. Notice that $v_{38}$ is "compatible" with $v_{32}$, which is the only complex state that is an ancestor of $v_{38}$, and hence, $\operatorname{Status}\left(v_{32}\right)$ is not changed (to incomplete).

Observe that the assertion $b:\langle\sigma\rangle\left\langle A_{3}\right\rangle \neg p$ is not $\diamond$-realizable at $v_{32}$ w.r.t. $v_{32}$. Hence, applying (close), Status $\left(v_{32}\right)$ is first changed to closed-wrt $\left(\left\{v_{32}\right\}\right)$ and then to closed, after that $\operatorname{Status}\left(v_{30}\right)$ is also changed to closed.

Applying $\left(\diamond_{?}\right), v_{31}$ is connected to a new simple non-state $v_{39}$ with

$$
\operatorname{Label}\left(v_{39}\right)=\left\{b:[\sigma]\left[A_{1}\right] p, b: p, \sigma(b, b), b: b, b:\left\langle A_{3}\right\rangle \neg p\right\} .
$$

Notice that $b:\left\langle A_{3}\right\rangle \neg p \in \operatorname{Reduced}\left(v_{39}\right)$. Applying (form-state), $v_{39}$ is connected to a new complex state $v_{40}$ with $\operatorname{Label}\left(v_{40}\right)=\operatorname{Label}\left(v_{39}\right)$. Applying (trans), Status $\left(v_{40}\right)$ is changed to expanded (without being connected to any nodes).

Observe that the assertion $b:\left\langle A_{3}\right\rangle \neg p$ is not $\diamond$-realizable at $v_{40}$ w.r.t. $v_{40}$. Hence, applying (close), Status $\left(v_{40}\right)$ is first changed to closed-wrt $\left(\left\{v_{40}\right\}\right)$ and then to closed, after that the statuses of $v_{39}, v_{31}, v_{28}, v_{27}, v_{8}, v_{5}-v_{1}, \nu$ are changed to closed in subsequent steps. Since Status $(\nu)=$ closed, by Theorem 3.3, we conclude that the given $\mathrm{ABox} \Gamma$ is unsatisfiable.

\section{Proofs}

In this section, let $\Gamma$ be an ABox in NNF and $G=(V, E, \nu)$ an arbitrary $\mathcal{C}_{\mathrm{HPDL}^{-}}$ tableau for $\Gamma$.

\subsection{Complexity Analysis}

We define the length of a formula (resp. program or assertion) to be the number of occurrences of symbols in that formula (resp. program or assertion). We define the size of a set of formulas and assertions to be the sum of the lengths of its formulas and assertions.

Definition 5.1. The set of basic subformulas of $\Gamma$, denoted by $b s f(\Gamma)$, consists of all subformulas of $\Gamma$ and their negations in NNF. The set closure $_{0}(\Gamma)$ is defined to be the smallest extension of $\Gamma \cup b s f(\Gamma)$ such that:

1. if $[\alpha] \varphi \in b s f(\Gamma), q \in Q_{\mathbb{A}_{\alpha}}, \omega$ is of the form $\sigma$ or $\psi$ ? and occurs in $\alpha$, then $\left[\mathbb{A}_{\alpha}, q\right] \varphi$ and $[\omega]\left[\mathbb{A}_{\alpha}, q\right] \varphi$ belong to closure $_{0}(\Gamma)$;

2. if $\langle\alpha\rangle \varphi \in b s f(\Gamma), q \in Q_{\mathbb{A}_{\alpha}}, \omega$ is of the form $\sigma$ or $\psi$ ? and occurs in $\alpha$, then $\left\langle\mathbb{A}_{\alpha}, q\right\rangle \varphi$ and $\langle\omega\rangle\left\langle\mathbb{A}_{\alpha}, q\right\rangle \varphi$ belong to closure $_{0}(\Gamma)$;

3. if $\varphi \in$ closure $_{0}(\Gamma)$ and $a$ is a nominal occurring in $\Gamma$, then $a: \varphi \in \operatorname{closure}_{0}(\Gamma)$.

The set $\operatorname{closure}(\Gamma)$ is defined to be the smallest extension of closure $_{0}(\Gamma)$ such that, if $\xi \in \operatorname{closure}(\Gamma)$, both nominals $a$ and $b$ occur in $\Gamma$, and $\xi^{\prime}$ is obtained from $\xi$ by replacing every occurrence of $b$ with $a$, including the ones in automata of modal operators, then $\xi^{\prime} \in \operatorname{closure}(\Gamma)$. 
Lemma 5.2. Let $n$ be the size of $\Gamma$. Then, $\mid$ closure $_{0}(\Gamma) \mid=O\left(n^{4}\right)$ and $\mid$ closure $(\Gamma) \mid=$ $O\left(2^{f(n)}\right)$ for some polynomial $f(\cdot)$.

Proof. The cardinality of $b s f(\Gamma)$ is of rank $O(n)$. Consider the construction of closure $_{0}(\Gamma)$ by starting from $b s f(\Gamma)$. Applying the first and second rules, we add $O\left(n^{3}\right)$ formulas to closure $_{0}(\Gamma)$ (note that the number of states of an automaton $\mathbb{A}_{\alpha}$ is linear in the length of $\alpha$ ). After that, applying the third rule, we add $O\left(n^{4}\right)$ assertions to closure $_{0}(\Gamma)$. Therefore, $\mid$ closure $_{0}(\Gamma) \mid=O\left(n^{4}\right)$. The second assertion of the lemma clearly follows.

Let $u$ be a complex node of $G$. For a formula/assertion $\xi$, by $\operatorname{NomRepl}(u)(\xi)$ we denote the formula/assertion obtained from $\xi$ by replacing every nominal $a$ with $\operatorname{NomRepl}(u)(a)$, including the ones in automata of modal operators. For a set $X$ of formulas/assertions, we define $\operatorname{NomRepl}(u)(X)=\{\operatorname{NomRepl}(u)(\xi) \mid \xi \in X\}$.

Lemma 5.3. Formulas and assertions used for the construction of any $\mathcal{C}_{\mathrm{HPDL}}$-tableau for $\Gamma$ belong to closure $(\Gamma)$. Furthermore, for every node $v$ of $G$, the cardinality of FullLabel $(v)$ is polynomial in the size of $\Gamma$.

Proof. The first assertion is clear. The second one follows from Lemma 5.2 and the observations: if $u$ is a complex node of $G$, then $\operatorname{Label}(u) \cup \operatorname{Reduced}(u) \subseteq$

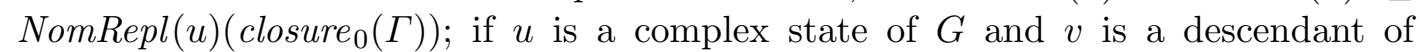
$u$, then FullLabel $(v) \subseteq \operatorname{NomRepl}(u)\left(\right.$ closure $\left._{0}(\Gamma)\right)$.

Corollary 5.4. Let $n$ be the size of $\Gamma$. Then, the number of nodes of $G$ is (at most) exponential in $n$. Consequently, $G$ can be constructed in exponential time in $n$.

Proof. The first assertion holds because $G$ is constructed using global caching and due the facts stated by Lemma 5.3 and the second assertion of Lemma 5.2. For the second assertion, just observe that each complex non-state may be re-expanded at most once.

\subsection{Soundness}

Our proof of soundness of the $\mathcal{C}_{\mathrm{HPDL}}$-tableau system relies on the notion of marking defined below.

Definition 5.5. Let $\mathcal{M}$ be a finitely branching Kripke model of $\Gamma$ and let $u$ be a complex state of $G$ such that Status $(u) \neq$ incomplete and $\mathcal{M} \models$ FullLabel $(u)$. Let $\mathcal{O}^{\prime}=\{a \in \mathcal{O} \mid \operatorname{NomRepl}(u)(a)=a\}$ and $V^{\prime}=\{v \in V \mid \operatorname{SType}(v)=$ simple $\}$. A marking of $G$ w.r.t. $\mathcal{M}$ and $u$ is a function $f: O^{\prime} \cup V^{\prime} \rightarrow P\left(\Delta^{\mathcal{M}}\right)$ with the intention that, for $x \in O^{\prime} \cup V^{\prime}, f(x)$ is the set of states of $\mathcal{M}$ that "correspond" to $x$. It is defined to be the limit resulted from the following construction:

- for each $a \in \mathcal{O}^{\prime}$, set $f(a):=\left\{a^{\mathcal{M}}\right\}$;

- for each $v \in V^{\prime}$, set $f(v):=\emptyset$;

- initialize $U$ to a queue containing all the pairs $\left(a, a^{\mathcal{M}}\right)$ for $a \in \mathcal{O}^{\prime}$ (in any order);

- while $U \neq \emptyset$, do:

- extract a pair $(x, y)$ from $U$;

- if $x \in \mathcal{O}^{\prime}$, then: 
* for every $v \in V^{\prime}$ such that $(u, v) \in E$, every $x:\langle\sigma\rangle \varphi \in \operatorname{ELabels}(u, v)$, and every $z \in(\operatorname{Label}(v))^{\mathcal{M}}$ such that $(y, z) \in \sigma^{\mathcal{M}}$, add $z$ to $f(v)$ and $(v, z)$ to $U$;

- else if Type $(x)=$ state, then:

* for every $v \in V^{\prime}$ such that $(x, v) \in E$, every $\langle\sigma\rangle \varphi \in \operatorname{ELabels}(x, v)$, and every $z \in(\operatorname{Label}(v))^{\mathcal{M}}$ such that $(y, z) \in \sigma^{\mathcal{M}}$, add $z$ to $f(v)$ and $(v, z)$ to $U$;

- else if there exists $a \in \operatorname{Label}(x)$, then:

* add $y$ to $f(a)$ and $(a, y)$ to $U$;

- else:

* for every $v \in V^{\prime}$ such that $(x, v) \in E$ and $y \in(\operatorname{Label}(v))^{\mathcal{M}}$, add $y$ to $f(v)$ and $(v, y)$ to $U$.

Lemma 5.6. Every path consisting of only non-states in $G$ is finite.

Proof. This lemma follows from the following observations:

- If a non-state $w$ is a successor of a non-state $v$ then $\operatorname{Reduced}(w) \supset \operatorname{Reduced}(v)$ or FullLabel $(w) \supset$ FullLabel $(v)$ or the number of nominals occurring in Label $(w)$ is smaller than the number of nominals occurring in Label $(v)$.

- For any node $w$, Reduced $(w)$ and FullLabel $(w)$ are subsets of the finite set closure $(\Gamma)$.

Lemma 5.7. Let $\mathcal{M}$ be a finitely branching Kripke model of $\Gamma$ and $u$ a complex state of $G$ such that Status $(u) \neq$ incomplete and $\mathcal{M} \models \operatorname{FullLabel}(u)$. Then Status $(u) \neq$ closed.

Proof. Let $f: O^{\prime} \cup V^{\prime} \rightarrow P\left(\Delta^{\mathcal{M}}\right)$ a marking of $G$ w.r.t. $\mathcal{M}$ and $u$, where $\mathcal{O}^{\prime}=\{a \in \mathcal{O} \mid$ $\operatorname{NomRepl}(u)(a)=a\}$ and $V^{\prime}=\{v \in V \mid \operatorname{SType}(v)=\operatorname{simple}\}$. Let $V^{\prime \prime}=\{u\} \cup\{w \in$ $\left.V^{\prime} \mid f(w) \neq \emptyset\right\}$. We prove that, if the status of a node $v \in V$ is changed to closed or closed-wrt ${ }_{+}(u)$, then $v \notin V^{\prime \prime}$, by induction on that moment.

Recall that $\mathcal{M} \models$ FullLabel $(u)$ and observe that, if $w \in V^{\prime}$ and $z \in f(w)$, then $z \in(\operatorname{Label}(w))^{\mathcal{M}}$ and thus $z \in(\text { FullLabel }(w))^{\mathcal{M}}$. Hence, if $w \in V^{\prime \prime}$, then FullLabel $(w)$ is satisfiable.

If $\operatorname{Status}(v)$ is changed to closed by the rule (close) because there exists $o: \perp \in$ Label $(v)$ or $a: \neg a \in \operatorname{Label}(v)$ or $\{\xi, \bar{\xi}\} \subseteq$ FullLabel $(v)$, then FullLabel $(v)$ is unsatisfiable and hence $v \notin V^{\prime \prime}$.

If $v \in V^{\prime \prime}$ and $\operatorname{Status}(v)$ was changed to closed by the rule (close) because $\operatorname{Status}(v)=$ closed-wrt ${ }_{+}(v)$, then $v$ must be a complex state and thus $v=u$, and by the inductive assumption, $v \notin V^{\prime \prime}$, a contradiction.

Consider the case when Status $(v)$ is changed to closed-wrt $(u)$ by the rule (nominal) and, for the sake of contradiction, assume that $v \in V^{\prime \prime}$. Thus, $v \in V^{\prime}$ and $f(v) \neq \emptyset$. Hence, FullLabel $(v)$ is satisfied at a state in $\mathcal{M}$, in particular, $\mathcal{M} \models \xi$ (where $\xi$ is the assertion mentioned in the rule (nominal)), which contradicts the facts that $\bar{\xi} \in \operatorname{FullLabel}(u)$ and $\mathcal{M} \models \operatorname{FullLabel}(u)$.

Observe that, if $w \in V^{\prime \prime}$, Status $(w) \notin$ \{unexpanded, closed, blocked, closed-wrt $(\ldots)\}$ and Type $(w)=$ non-state, then $w$ must have a successor belonging to $V^{\prime \prime}$. Hence, if Status $(v)$ is changed to closed or closed-wrt ${ }_{+}(u)$ by the instruction 2 of the rule (close), then, by the inductive assumption, it follows that $v \notin V^{\prime \prime}$. 
Similarly, if $w \in V^{\prime \prime}$, Status $(w) \notin\{$ unexpanded, closed, incomplete $\}$ and Type $(w)=$ state, then all successors of $w$ must belong to $V^{\prime \prime}$. Hence, if Status $(v)$ is changed to closed or closed-wrt ${ }_{+}(u)$ by the instruction 3 of the rule (close), then, by the inductive assumption, it follows that $v \notin V^{\prime \prime}$.

There remains the case when $\operatorname{Status}(v)$ is changed to closed-wrt $_{+}(u)$ by the instruction $1 \mathrm{~b}$ of the rule (close). For this case, it is sufficient to prove that:

1. every assertion of the form $a:\langle A, q\rangle \varphi$ or $a:\langle\omega\rangle\langle A, q\rangle \varphi$ in FullLabel $(u)$ is $\diamond$ realizable at $u$ w.r.t. $u$,

2. if $w \in V^{\prime}$ and $f(w) \neq \emptyset$, then every formula of the form $\langle A, q\rangle \varphi$ or $\langle\omega\rangle\langle A, q\rangle \varphi$ in FullLabel $(w)$ is $\diamond$-realizable at $w$ w.r.t. $u$.

Consider the second assertion and suppose that $w \in V^{\prime}, z \in f(w), \xi \in$ FullLabel $(w)$ and $\xi$ is of the form $\langle A, q\rangle \varphi$ or $\langle\omega\rangle\langle A, q\rangle \varphi$. We have that $z \in$ $(\operatorname{Label}(w))^{\mathcal{M}}$. Consider the case when $\xi=\langle A, q\rangle \varphi$ (the other case is similar and omitted). Since $z \in(\operatorname{Label}(w))^{\mathcal{M}}$, there exist $z_{0}, \ldots, z_{k} \in \Delta^{\mathcal{M}}$ and a word $\omega_{1} \ldots \omega_{k}$ accepted by $(A, q)$ such that $z_{0}=z, z_{k} \in \varphi^{\mathcal{M}}$ and, for each $1 \leq i \leq k$, if $\omega_{i} \in \Sigma$ then $\left(z_{i-1}, z_{i}\right) \in \omega_{i}^{\mathcal{M}}$, else $\omega_{i}=\left(\psi_{i}\right.$ ? $)$ for some $\psi_{i}$ and $z_{i-1}=z_{i}$ and $z_{i} \in \psi_{i}^{\mathcal{M}}$. Such a realization (satisfaction) of $\xi$ at $z$ in $\mathcal{M}$ is reflected by a sequence $\left(w_{0}, \xi_{0}\right), \ldots,\left(w_{h}, \xi_{h}\right)$ such that $w_{0}, \ldots, w_{h} \in V^{\prime \prime}, w_{0}=w, \xi_{0}=\xi, \xi_{i}$ is of the form $o_{i}:\left\langle A, q_{i}\right\rangle \varphi$ or $o_{i}:\left\langle\omega_{i}^{\prime}\right\rangle\left\langle A, q_{i}\right\rangle \varphi$ (for $0 \leq i \leq h$ ), $\xi_{h}$ is $\diamond$-realizable at $w_{h}$ w.r.t. $u$ by the condition 2a or 2d of Definition 3.2, and each $\xi_{i}$ with $0 \leq i<h$ is $\diamond$-realizable at $w_{i}$ w.r.t. $u$ because $\xi_{i+1}$ is $\diamond$-realizable at $w_{i+1}$ w.r.t. $u$, due to a condition among 2b, 2c, 2e 2il of Definition 3.2, For this claim, we use the inductive assumption and the fact that every path consisting of only non-states in $G$ is finite (Lemma [5.6). As a consequence, $\xi$ is $\diamond$-realizable at $w$ w.r.t. $u$.

The first assertion in the above list can be proved analogously.

Corollary 5.8 (Soundness). Let $\Gamma$ be an $A B o x$ in $N N F$ and $G=(V, E, \nu)$ an arbitrary $\mathcal{C}_{\mathrm{HPDL}}$-tableau for $\Gamma$. If $\Gamma$ is satisfiable, then Status $(\nu) \neq$ closed.

Proof. Assume that $\Gamma$ is satisfiable. It is well known that, if an ABox (in HPDL) is satisfiable, then it has a finitely-branching Kripke model. Let $\mathcal{M}$ be a finitelybranching Kripke model of $\Gamma$. Since $\operatorname{Label}(\nu)=\Gamma$ and $\mathcal{M} \models \Gamma$, there exists a complex state $u$ of $G$ such that Status $(u) \neq$ incomplete and $\mathcal{M} \models$ FullLabel $(u)$. By Lemma 5.7, Status $(u) \neq$ closed. This implies that $\operatorname{Status}(\nu) \neq$ closed.

\subsection{Completeness}

In this subsection, assume that $\operatorname{Status}(\nu) \neq$ closed (where $\nu$ is the root of $G$ ). We prove that $\Gamma$ is satisfiable by constructing a model graph $G^{\prime}$ of $G$ and a Kripke model $\mathcal{M}$ that corresponds to $G^{\prime}$.

Since $\operatorname{Status}(\nu) \neq$ closed, there exists a complex state $u$ of $G$ such that $\operatorname{Status}(u) \notin$ $\{$ closed, incomplete $\}$. In this subsection, we fix such a $u$. We also fix a sequential process of marking assertions/formulas of the form $o:\langle A, q\rangle \varphi$ or $o:\langle\omega\rangle\langle A, q\rangle \varphi$ in the full labels of nodes of $G$ as $\diamond$-realizable w.r.t. $u$. For $v \in V$ and $\xi \in \operatorname{FullLabel}(v)$ of one of these forms, let TimeStampDR $(\xi, v, u)$ denote the moment at which $\xi$ is marked as $\diamond$-realizable at $v$ w.r.t. $u$. In the other case, $\operatorname{TimeStampDR}(\xi, v, u)$ is undefined. 
Definition 5.9. Let $v$ be a descendant of $u$ with Status $(v) \notin\{$ closed, closed-wrt $\left.{ }_{+}(u)\right\}$ and let $\xi \in$ FullLabel $(v)$ be of the form $o:\langle A, q\rangle \varphi$ or $o:\langle\omega\rangle\langle A, q\rangle \varphi$. A $\diamond$-realization of $\xi$ at $v$ w.r.t. $u$ is a sequence $\left(v_{0}, \xi_{0}\right), \ldots,\left(v_{k+1}, \xi_{k+1}\right)$ such that:

$-k \geq 0, v_{0}=v$ and $\xi_{0}=\xi$,

- for each $0 \leq i \leq k, \xi_{i} \in$ FullLabel $\left(v_{i}\right), \xi_{i}$ is of the form $o_{i}:\left\langle A, q_{i}\right\rangle \varphi$ or $o_{i}:\left\langle\omega_{i}\right\rangle\left\langle A, q_{i}\right\rangle \varphi$ and is $\diamond$-realizable at $v_{i}$ w.r.t. $u$,

- for each $0 \leq i<k$, TimeStampDR $\left(\xi_{i}, v_{i}, u\right)>\operatorname{TimeStampDR}\left(\xi_{i+1}, v_{i+1}, u\right)$ and $\xi_{i}$ was marked at the moment TimeStampDR $\left(\xi_{i}, v_{i}, u\right)$ as $\diamond$-realizable at $v_{i}$ w.r.t. $u$ due to the $\diamond$-realizability of $\xi_{i+1}$ at $v_{i+1}$ w.r.t. $u$ according to the rules specified by the conditions 2b, 2c, 2e 2il of Definition 3.2,

- one of the following two conditions holds:

- $\xi_{k}$ was marked at the moment TimeStampDR $\left(\xi_{k}, v_{k}, u\right)$ as $\diamond$-realizable at $v_{k}$ w.r.t. $u$ due to the rule specified by the condition 2d of Definition 3.2, $v_{k}$ was expanded by the tableau rule $\left(\langle\mathrm{A}\rangle_{f}\right)$ with $\xi_{k}=o_{k}:\left\langle A, q_{k}\right\rangle \varphi$ being the principal formula (having $q_{k} \in F_{A}$ ), $v_{k+1}$ is the successor of $v_{k}$ whose label is obtained from Label $\left(v_{k}\right)$ by replacing $\xi_{k}$ with $\xi_{k+1}=o_{k+1}: \varphi$, and $\operatorname{Status}\left(v_{k+1}\right) \notin$ $\left\{\right.$ closed, closed-wrt $\left.{ }_{+}(u)\right\}$;

- $\xi_{k}$ was marked at the moment TimeStampDR $\left(\xi_{k}, v_{k}, u\right)$ as $\diamond$-realizable at $v_{k}$ w.r.t. $u$ due to the rule specified by the condition $2 \mathrm{a}$ of Definition 3.2 , $\xi_{k}=o_{k}:\left\langle A, q_{k}\right\rangle \varphi, q_{k} \in F_{A}, o_{k+1}: \varphi \in$ FullLabel $\left(v_{k}\right), v_{k+1}=v_{k}$ and $\xi_{k+1}=$ $o_{k+1}: \varphi$.

Lemma 5.10. If $v$ is a descendant of $u$ with Status $(v) \notin\left\{\right.$ closed, closed-wrt $\left.t_{+}(u)\right\}$, then every $\xi \in$ FullLabel $(v)$ of the form $o:\langle A, q\rangle \varphi$ or $o:\langle\omega\rangle\langle A, q\rangle \varphi$ has a $\diamond$-realization at $v$ w.r.t. $u$.

This lemma clearly holds, because $\xi$ has a $\diamond$-realization at $v$ w.r.t. $u$ iff it is $\diamond$-realizable at $v$ w.r.t. $u$.

Definition 5.11. Let $v$ be a descendant of $u$ such that it is a simple non-state and Status $(v) \notin\left\{\right.$ closed, closed-wrt $\left.t_{+}(u)\right\}$. A saturation path of $v$ w.r.t. $u$ is a sequence $v_{0}$, $v_{1}, \ldots, v_{k}$ of nodes of $G$, with $v_{0}=v$ and $k \geq 1$, such that:

- Status $\left(v_{i}\right) \notin\left\{\right.$ closed, closed-wrt $\left.{ }_{+}(u)\right\}$ for all $0 \leq i \leq k$,

- Type $\left(v_{i}\right)=$ non-state for all $0 \leq i<k$ and Type $\left(v_{k}\right)=$ state,

$-\left(v_{i}, v_{i+1}\right) \in E$ for all $0 \leq i<k-1$,

- if there exists $a \in \operatorname{Label}\left(v_{k-1}\right)$, then $v_{k}=u$, else $\left(v_{k-1}, v_{k}\right) \in E$.

By Lemma 5.6, each saturation path of $v$ w.r.t. $u$ is finite. Furthermore, if $v_{i}$ is a simple non-state with $\operatorname{Status}\left(v_{i}\right) \notin\{$ closed, closed-wrt $+(u)\}$ and Label $\left(v_{i}\right)$ does not contain any nominal, then $v_{i}$ has a successor $v_{i+1}$ with $\operatorname{Status}\left(v_{i+1}\right) \notin\{$ closed, closed-wrt $\left.t_{+}(u)\right\}$. Therefore, we have the following lemma.

Lemma 5.12. If $v$ is a descendant of $u$ such that it is a simple non-state and Status $(v) \notin\left\{\right.$ closed, closed-wrt $\left.{ }_{+}(u)\right\}$, then it has at least one saturation path w.r.t. $u$.

Definition 5.13. A model graph of $G$ w.r.t. $u$ is a structure $G^{\prime}=$ $\left(V^{\prime}, E^{\prime}\right.$, Label $^{\prime}$, ELabels $\left.{ }^{\prime}\right)$ constructed as follows, where $V^{\prime} \subseteq \mathcal{O} \cup\{v \in V \mid$ SType $(v)=$ simple and Type $(v)=$ state $\}, E^{\prime} \subseteq V^{\prime} \times V^{\prime}$, ELabels ${ }^{\prime}: E^{\prime} \rightarrow P(\Sigma)$, and for $x \in V^{\prime}$, $\operatorname{Label}^{\prime}(x)$ is a set of formulas: 
1. $V^{\prime}:=\{a \in \mathcal{O} \mid \operatorname{NomRepl}(u)(a)=a\}$;

2. $E^{\prime}:=\{(a, b) \mid$ there exists $\sigma(a, b) \in \operatorname{Label}(u)\}$;

3. for each $a \in V^{\prime}, \operatorname{Label}^{\prime}(a):=\{\varphi \mid a: \varphi \in$ FullLabel $(u)\} \cup\{a\}$;

4. for each $(a, b) \in E^{\prime}, \operatorname{ELabels}(a, b):=\{\sigma \mid \sigma(a, b) \in \operatorname{Label}(u)\}$;

5. realized $:=\emptyset$;

6. while there exist $x \in V^{\prime}$ and $\varphi=\langle\sigma\rangle\langle A, q\rangle \psi \in \operatorname{Label}^{\prime}(x)$ such that $(x, \varphi) \notin$ realized, do:

(a) if $x \in V$, then let $\left(v_{0}, \xi_{0}\right), \ldots,\left(v_{k+1}, \xi_{k+1}\right)$ be a $\diamond$-realization of $\varphi$ at $x$ w.r.t. $u$, else let $\left(v_{0}, \xi_{0}\right), \ldots,\left(v_{k+1}, \xi_{k+1}\right)$ be a $\diamond$-realization of $x: \varphi$ at $u$ w.r.t. $u$;

(b) if Type $\left(v_{k+1}\right)=$ state, then let $l=1$, else let $v_{k+1}, \ldots, v_{k+l}$ be a saturation path of $v_{k+1}$ w.r.t. $u$;

(c) let $i_{1}, \ldots, i_{h}$ be all the indices such that $0<i_{1}<\ldots<i_{h}=k+l$, Type $\left(v_{i_{j}}\right)=$ state for $1 \leq j \leq h$, and for every $1 \leq j<h$, if $v_{i_{j}}=u$, then $\xi_{i_{j}}$ is of the form $a_{j}:\left\langle\sigma_{j}\right\rangle\left\langle A, q_{i_{j}}\right\rangle \psi$, else $\xi_{i_{j}}$ is of the form $\left\langle\sigma_{j}\right\rangle\left\langle A, q_{i_{j}}\right\rangle \psi$;

(d) if $v_{i_{h}}=u$, then let $a_{h}$ be a nominal such that $a_{h} \in \operatorname{Label}\left(v_{i}\right)$, where $i$ is the greatest index such that $i<i_{h}$ and $v_{i} \neq u$;

(e) $x_{0}:=x, \sigma_{0}:=\sigma$;

(f) for each $j$ from 1 to $h$, do:

i. if $v_{i_{j}} \neq u$, then:

$-x_{j}:=v_{i_{j}}$;

- if $x_{j} \notin V^{\prime}$, then add $x_{j}$ to $V^{\prime}$ and set $\operatorname{Label}^{\prime}\left(x_{j}\right):=\operatorname{FullLabel}\left(v_{i_{j}-1}\right)$;

- else $\operatorname{Label}^{\prime}\left(x_{j}\right):=\operatorname{Label}^{\prime}\left(x_{j}\right) \cup \operatorname{Reduced}\left(v_{i_{j}-1}\right)$;

- add $\left(x_{j-1}, x_{j}\right)$ to $E^{\prime}$ and $\sigma_{j-1}$ to ELabels $\left(x_{j-1}, x_{j}\right)$;

ii. else: $x_{j}:=a_{j}$, add $\left(x_{j-1}, x_{j}\right)$ to $E^{\prime}$ and $\sigma_{j-1}$ to ELabels $\left(x_{j-1}, x_{j}\right)$;

(g) add $(x, \varphi)$ to realized.

As invariants of the "while" loop in the above construction, we have that:

- if $x \in V^{\prime}$, then Status $(x) \notin\left\{\right.$ closed, closed-wrt $\left.{ }_{+}(u)\right\}$;

- the "let" instruction at the step 6a is well-defined due to Lemma 5.10,

- the "let" instruction at the step 6 b is well-defined due to Lemma 5.12.

Also observe that:

- the "let" instruction at the step $6 \mathrm{c}$ is well-defined; it specifies not only $i_{1}, \ldots, i_{h}$, but also $\sigma_{j}$ for all $1 \leq j<h$, and $a_{j}$ for all $1 \leq j<h$ such that $v_{i_{j}}=u$;

- since $G$ is finite (by Corollary 5.4), the "while" loop terminates and $G^{\prime}$ is finite.

The following lemma states that a model graph is similar to a Hintikka structure. It directly follows from the constructions of $G$ and $G^{\prime}$.

Lemma 5.14. Let $G^{\prime}=\left(V^{\prime}, E^{\prime}\right.$, Label $^{\prime}$, ELabels $\left.{ }^{\prime}\right)$ be a model graph of $G$ w.r.t. $u$. Then, for every $x \in V^{\prime}$ and every $\varphi \in \operatorname{Label}^{\prime}(x)$ :

1. $\perp \notin \operatorname{Label}^{\prime}(x)$ and $\bar{\varphi} \notin \operatorname{Label}^{\prime}(x)$,

2. if $\varphi=\psi \wedge \chi$, then $\{\psi, \chi\} \subset \operatorname{Label}^{\prime}(x)$,

3. if $\varphi=\psi \vee \chi$, then $\psi \in \operatorname{Label}^{\prime}(x)$ or $\chi \in \operatorname{Label}^{\prime}(x)$,

4. if $\varphi=a$, then $x=a$,

5. if $\varphi=[\alpha] \psi, \alpha \notin \Sigma, \alpha$ is not a test and $I_{\mathbb{A}_{\alpha}}=\left\{q_{1}, \ldots, q_{k}\right\}$, then $\left\{\left[\mathbb{A}_{\sigma}, q_{1}\right] \psi, \ldots,\left[\mathbb{A}_{\sigma}, q_{k}\right] \psi\right\} \subset$ Label $^{\prime}(x)$, 
6. if $\varphi=[A, q] \psi$ and $\delta_{A}(q)=\left\{\left(\omega_{1}, q_{1}\right), \ldots,\left(\omega_{k}, q_{k}\right)\right\}$, then $\left\{\left[\omega_{1}\right]\left[A, q_{1}\right] \psi, \ldots,\left[\omega_{k}\right]\left[A, q_{k}\right] \psi\right\} \subset \operatorname{Label}^{\prime}(x)$,

7. if $\varphi=[A, q] \psi$ and $q \in F_{A}$, then $\psi \in \operatorname{Label}^{\prime}(x)$,

8. if $\varphi=[\chi ?] \psi$, then $\bar{\chi} \in \operatorname{Label}^{\prime}(x)$ or $\psi \in \operatorname{Label}^{\prime}(x)$,

9. if $\varphi=[\sigma] \psi,(x, y) \in E^{\prime}$ and $\sigma \in$ ELabels $^{\prime}(x, y)$, then $\psi \in$ Label $^{\prime}(y)$,

10. if $\varphi=\langle\alpha\rangle \psi, \alpha \notin \Sigma, \alpha$ is not a test and $I_{\mathbb{A}_{\alpha}}=\left\{q_{1}, \ldots, q_{k}\right\}$, then $\left\{\left\langle\mathbb{A}_{\alpha}, q_{1}\right\rangle \psi, \ldots,\left\langle\mathbb{A}_{\alpha}, q_{k}\right\rangle \psi\right\} \cap$ Label $^{\prime}(x) \neq \emptyset$,

11. if $\varphi=\langle\chi ?\rangle \psi$, then $\{\chi, \psi\} \subseteq$ Label $^{\prime}(x)$,

12. if $\varphi=\langle\sigma\rangle \psi$, then there exists $y$ such that $(x, y) \in E^{\prime}, \sigma \in$ ELabels $^{\prime}(x, y)$ and $\psi \in \operatorname{Label}^{\prime}(y)$,

13. if $\varphi=\langle A, q\rangle \psi$, then there exist an accepting run $q_{0}, \ldots, q_{k}$ of the automaton $(A, q)$ on a word $\omega_{1} \ldots \omega_{k}$ (with $q_{0}=q$ and $q_{k} \in F_{A}$ ) and a sequence $x_{0}, \ldots, x_{k}$ of nodes of $G^{\prime}$ such that $x_{0}=x, \psi \in \operatorname{Label}^{\prime}\left(x_{k}\right)$ and, for each $1 \leq i \leq k$, if $\omega_{i}=\left(\chi_{i}\right.$ ?), then $x_{i}=x_{i-1}$ and $\left\{\chi_{i},\left\langle A, q_{i}\right\rangle \psi\right\} \subseteq \operatorname{Label}^{\prime}\left(x_{i}\right)$, else $\left(x_{i-1}, x_{i}\right) \in E^{\prime}$, $\omega_{i} \in$ ELabels $^{\prime}\left(x_{i-1}, x_{i}\right)$ and $\left\langle A, q_{i}\right\rangle \psi \in \operatorname{Label}^{\prime}\left(x_{i}\right)$.

Definition 5.15. Let $G^{\prime}=\left(V^{\prime}, E^{\prime}\right.$, Label $^{\prime}$, ELabels $)$ be a model graph of $G$ w.r.t. $u$. A Kripke model $\mathcal{M}$ corresponds to $G^{\prime}$ w.r.t. $u$ if:

$-\Delta^{\mathcal{M}}=V^{\prime}$

$-p^{\mathcal{M}}=\left\{x \in V^{\prime} \mid p \in\right.$ Label $\left.^{\prime}(x)\right\}$ for $p \in \mathcal{P} \mathcal{R O} \mathcal{O}$,

- $\sigma^{\mathcal{M}}=\left\{(x, y) \in E^{\prime} \mid \sigma \in\right.$ ELabels $\left.^{\prime}(x, y)\right\}$ for $\sigma \in \Sigma$,

$-a^{\mathcal{M}}=\operatorname{NomRepl}(u)(a)$ for $a \in \mathcal{O}$ with $\operatorname{NomRepl}(u)(a)$ specified.

Clearly, there exist Kripke models corresponding to $G^{\prime}$ w.r.t. $u$. They differ from each other only in interpreting nominals $a$ with $\operatorname{NomRepl}(u)(a)$ unspecified.

Lemma 5.16. Let $G^{\prime}=\left(V^{\prime}, E^{\prime}\right.$, Label $^{\prime}$, ELabels $\left.{ }^{\prime}\right)$ be a model graph of $G$ w.r.t. u and $\mathcal{M}$ a Kripke model corresponding to $G^{\prime}$ w.r.t. u. Then:

1. for every $x \in V^{\prime}$ and every $\varphi \in \operatorname{Label}^{\prime}(x)$, we have $x \in \varphi^{\mathcal{M}}$,

2. $\mathcal{M} \models$ FullLabel $(u)$,

3. $\mathcal{M} \models \operatorname{Label}(\nu)$.

Proof. The first assertion can be proved in a straightforward way by induction on the structure of $\varphi$ using Lemma 5.14. The second assertion follows from the first one, the initialization of the construction of $G^{\prime}$ and the interpretation of nominals in $\mathcal{M}$. The third assertion follows from the second one. Namely, there exists a path $v_{0}, \ldots, v_{k}$ in $G$ such that $v_{0}=\nu$ and $v_{k}=u$, and by the applied tableau rules, for every $i$ from $k-1$ down to $0, \mathcal{M} \models$ FullLabel $\left(v_{i}\right)$ follows from $\mathcal{M} \models \operatorname{FullLabel}\left(v_{i+1}\right)$.

Corollary 5.17 (Completeness). Let $\Gamma$ be an $A B o x$ in $N N F$ and $G=(V, E, \nu)$ an arbitrary $\mathcal{C}_{\mathrm{HPDL}}$-tableau for $\Gamma$. If Status $(\nu) \neq$ closed, then $\Gamma$ is satisfiable.

This corollary follows from the third assertion of Lemma $5.16($ since Label $(\nu)=\Gamma)$.

\section{Concluding Remarks}

We have given the first direct tableau procedure with the ExPTIME complexity for deciding HPDL and proved that it is sound and complete. The procedure uses global caching, a technique that not only guarantees the ExPTIme complexity, but also 
increases efficiency. As HPDL can be used as a description logic for representing and reasoning about terminological knowledge, our procedure is useful for practical applications.

In our decision procedure, any expansion strategy can be used for constructing a tableau. One can give the instruction 1(a) of the rule (close) the highest priority and give unary static rules a higher priority than for non-unary static rules. One may choose the depth-first expansion strategy, globally cache only simple nodes, keep complex nodes only for the current path of complex nodes, and is still guaranteed to have the EXPTime complexity for the algorithm. Checking fulfillment of eventualities can be done on-the-fly as in [1] or periodically for the whole graph or at special moments for subgraphs (for example, when the subgraph rooted at a node has been "fully expanded" and no $\diamond$-realization goes out from that subgraph).

Our decision procedure has been designed to simplify the presentation and leaves space for improvement. It has been implemented for the TGC2 system [12] with various optimizations. For example, a sequence of expansions by unary static rules is done in one step to eliminate intermediate non-states with only one successor, automata in modal operators are minimized, and different control strategies (for expanding the constructed tableau) are mixed. As TGC2 aims to allow efficient automated reasoning in a large class of modal and description logics, its implementation is time-consuming. A few intended important optimization techniques like propagation of unsatisfiability cores or compacting ABoxes by using bisimilarity were not implemented for TGC2 yet. In general, TGC2 still needs improvements, at least with respect to functionality. We refer the reader to [12] for more details about this system.

Our tableau method can be extended for Graded HPDL using the techniques from [13] and for Converse-HPDL using the techniques from [7]10]1].

\section{References}

1. P. Abate, R. Goré, and F. Widmann. An on-the-fly tableau-based decision procedure for PDLsatisfiability. Electronic Notes in Theoretical Computer Science, 231:191-209, 2009.

2. F. Baader and U. Sattler. An overview of tableau algorithms for description logics. Studia Logica, 69:5-40, 2001.

3. F. Donini and F. Massacci. EXPTime tableaux for $\mathcal{A L C}$. Art. Intelligence, 124:87-138, 2000.

4. B. Dunin-Kȩplicz, L.A. Nguyen, and A. Szałas. Converse-PDL with regular inclusion axioms: a framework for MAS logics. Journal of Applied Non-Classical Logics, 21(1):61-91, 2011.

5. M.J. Fischer and R.E. Ladner. Propositional dynamic logic of regular programs. J. Comput. Syst. Sci., 18(2):194-211, 1979.

6. R. Goré and L.A. Nguyen. ExpTime tableaux for $\mathcal{A L C}$ using sound global caching. J. Autom. Reasoning, 50(4):355-381, 2013.

7. R. Goré and F. Widmann. Optimal and cut-free tableaux for propositional dynamic logic with converse. In Proceedings of IJCAR 2010, volume 6173 of LNCS, pages 225-239. Springer, 2010.

8. D. Harel, D. Kozen, and J. Tiuryn. Dynamic Logic. MIT Press, 2000.

9. M. Kaminski and G. Smolka. A goal-directed decision procedure for hybrid PDL. J. Autom. Reasoning, 52(4):407-450, 2014.

10. L.A. Nguyen. Cut-free ExpTime tableaux for Converse-PDL extended with regular inclusion axioms. In Proc. of KES-AMSTA'2013, volume 252 of Frontiers in Artificial Intelligence and Applications, pages 235-244. IOS Press, 2013.

11. L.A. Nguyen. ExpTime tableaux with global state caching for the description logic $\mathcal{S H I O}$. Neurocomputing, 146:249-263, 2014.

12. L.A. Nguyen. Design of the tableau reasoner TGC2 for description logics. International Journal of Software Engineering and Knowledge Engineering, 26(8):1315-1333, 2016.

13. L.A. Nguyen. ExpTime tableaux with global caching for graded propositional dynamic logic. Fundam. Inform., 147(2-3):261-288, 2016. 
14. L.A. Nguyen and A. Szałas. Checking consistency of an ABox w.r.t. global assumptions in PDL. Fundamenta Informaticae, 102(1):97-113, 2010.

15. V.R. Pratt. A near-optimal method for reasoning about action. J. Comput. Syst. Sci., 20(2):231$254,1980$.

16. U. Sattler and M.Y. Vardi. The hybrid $\mu$-calculus. In Proceedings of IJCAR 2001, volume 2083 of $L N C S$, pages 76-91. Springer, 2001.

17. K. Schild. A correspondence theory for terminological logics: Preliminary report. In Proceedings of IJCAI'1991, pages 466-471, 1991. 